\title{
A Renormalizable Field Theory: The Massive Gross-Neveu Model in Two Dimensions
}

\author{
Joel Feldman ${ }^{1}$, J. Magnen ${ }^{2}$, V. Rivasseau ${ }^{2}$, and R. Sénéor ${ }^{2}$ \\ 1 Mathematics Department, University of British Columbia, Vancouver, B.C., Canada V6T 1Y4 \\ ${ }^{2}$ Centre de Physique Théorique, Ecole Polytechnique, F-91128 Palaiseau Cedex, France
}

\begin{abstract}
The Euclidean massive Gross-Neveu model in two dimensions is just renormalizable and asymptotically free. Thanks to the Pauli principle, bare perturbation theory with an ultra-violet cut-off (and the correct ansatz for the bare mass) is convergent in a disk, whose radius corresponds by asymptotic freedom to a small finite renormalized coupling constant. Therefore, the theory can be fully constructed in a perturbative way. It satisfies the O.S. axioms and is the Borel sum of the renormalized perturbation expansion of the model
\end{abstract}

\section{Introduction}

The Gross-Neveu (or Mitter-Weisz) models in two dimensions (in short the $\mathrm{GN}_{2}$ models) are among the simplest physical field theories which are asymptotically free, hence are among the most obvious "laboratories" to investigate the perturbative behavior of non-abelian gauge theories in four dimensions, and nonperturbative phenomena like spontaneous symmetry breaking $[1,2]$. They are models of $N$-component fermions (with $N \geqq 2$ ), with a quartic interaction; therefore their graphs are topologically the same as those of the familiar $\Phi^{4}$ bosonic theories.

Although the models discussed by Gross and Neveu were massless, and their paper [2] was mostly devoted to the discussion of chiral symmetry breaking and dynamical mass generation, the massive version (i.e., with non-zero bare mass) makes perfect sense. In contrast with the massless case these massive $\mathrm{GN}_{2}$ theories, while still asymptotically free [1], should have a purely perturbative content (by this we mean that all the physics of the model could be extracted from ordinary perturbation theory). Therefore, they are the simplest candidates for a rigorous construction of a renormalizable field theory, and it is slightly surprising that they were not often presented as such by "constructive field theory." (Of course these models are only two-dimensional; we remark also that the case $N=1$, which corresponds to the massive Thirring model has been solved by means of the sine Gordon transformation [3].) 
In this paper we construct the Schwinger functions of the models by "brute force", relying on the asymptotic freedom of the model and on the existence of a non-zero bare mass to get a small parameter of expansion. An outline of the construction can be found in [4] (although the bare mass flow is not correctly discussed there). In fact it is not harder than the construction of planar $-g \Phi_{4}^{4}$ field theories [5-7] and is certainly easier than the control of infrared $\Phi_{4}^{4}[8,9]$, since there is no "domination" or large fields problem. Its main interest is to prove on a "physical" example that asymptotic freedom is truly an effective tool, which like superrenormalizability provides rigorous control over the continuum limit of a quantum field theory. The recent construction of a hierarchical version of (nonplanar) $-g \Phi_{4}^{4}$ (but without physical positivity) [10] had the same motivation. All these results make us confident that asymptotic freedom will control in the same way the ultra-violet limit of four-dimensional non-abelian gauge theories, although the technical problems related to gauge invariance have not as yet been solved.

The organization of this paper is as follows: in Sect. 2 we introduce the model and the notation, we establish its perturbative renormalizability and we show, using Gram's inequality on determinants, that the bare unnormalized perturbation expansion of the model with infrared and ultraviolet cutoffs is absolutely convergent, no matter how large the bare coupling is. Therefore, the unnormalized, unrenormalized Schwinger functions in a finite volume, with finite ultra-violet cutoff, are entire functions of the bare coupling. Although the proof is extremely simple and certainly known to many experts on fermionic theories, we feel that this marvelous fact will never be over-emphasized.

In Sect. 3 we introduce first a single cluster and Mayer expansion. It allows us to perform the infinite-volume limit and to show that the normalized, unrenormalized Schwinger functions with an ultra-violet cutoff still have an absolutely convergent perturbation expansion for small enough bare coupling (depending on the cutoff). Since there are ultraviolet divergences in the theory, the radius of convergence shrinks to zero as the ultra-violet cutoff is removed. To push further the analysis, we introduce a phase-space expansion, with momentum slices $1, M$, $M^{2}, \ldots, M^{e}, M$ being a fixed integer, and cubes of corresponding size as in [9], and we repeat the cluster and Mayer expansion in each slice.

Now in Sect. 4 starting with a given bare coupling and the correct ansatz for the bare mass ${ }^{1}$, we can reshuffle the bare expansion into a "partly renormalized" expansion with effective coupling constants, masses and wave function constants which depend on the highest slice of momentum entering a vertex or flowing through a line. The transformation from bare to effective couplings is a renormalization group transformation, controlled by the first orders of perturbation theory. Let us remark at this point that for us "partly renormalized" expansions are more than a tool; since they lie at the right point between bare and renormalized expansions, we think that they should become the modern way of using perturbation theory (see also [5-7]). We call them PRPSE (partly renormalized phase space expansion).

1 We thank K. Gawedzki and A. Kupiainen for explaining the importance of this point to us 
In Sect. 5 we prove that this partly renormalized perturbation expansion is absolutely convergent, uniformly in the u.v. cutoff $M^{\varrho}$, provided the bare coupling $\lambda_{\varrho}$ is positive and chosen according to the formula obtained by retaining the first orders of perturbation theory:

$$
\lambda_{\varrho}(C)=f(\varrho, C)^{-1}, \quad \text { where } \quad f(\varrho, C) \equiv-\beta_{2}(\log M) \varrho+\left(\beta_{3} / \beta_{2}\right) \log \varrho+C
$$

$C$ being a large enough positive constant.

Moreover, as the ultra-violet cutoff is removed, the limit of the Schwinger functions which are the sums of this expansion does exist.

In Sect. 6 we investigate further the theory built in this way. We rewrite the partly renormalized expansion into an expansion in which the two-point subgraphs are "fully renormalized," and the four-point subgraphs are "partly renormalized;" therefore this expansion is expressed in terms of effective coupling constants and of renormalized mass and wave function constants. We prove that this expansion, with an u.v. cutoff, is still absolutely convergent, but now for any complex bare coupling in the disk of radius $f(\varrho, C)^{-1}$, as announced in [4]. This expansion, although of a somewhat mixed nature, is a beautiful tool, which could have been in fact taken as our starting point; it allows a particularly simple verification of the Osterwalder-Schrader axioms, and it allows also to identify the theory with the Borel sum of the (fully) renormalized expansion of the model, with, e.g., B.P.H.Z. subtractions at 0 external momenta (this expansion is expected to be divergent, due to the presence of ultra-violet renormalons).

Let us summarize what emerges at the end of our analysis as our recipe to compute the Gross-Neveu model in terms of Feynman amplitudes:

Take two values $C$ and $m$ ( $C$ large), and sum up all connected Feynman amplitudes of the theory on a lattice of mesh $M^{-\varrho}$ or with a momentum cutoff $M^{\varrho}$ of Pauli-Villars type and vertices $\lambda_{\varrho}$, where $\lambda_{\varrho}$ is defined by (1.1), and the two-point subgraphs are renormalized according to the BPHZ prescription (actually in this case this prescription is particularly simple: you don't even need to know about forests to use it since proper two point subgraphs never overlap). Although this was not obvious at first sight, tremendous cancellations occur at each order, so that the sum is absolutely convergent. Let now $\varrho$ tend to infinity; your sum converges to a continuum theory satisfying the axioms.

Finally, let us remark that our PRPSE, as well as the closely related formalism of [7], can be easily adapted to the construction of non-renormalisable theories, with fixed point close to the origin (see $[11,12])$. For instance the construction of the Gross-Neveu model with propagator $(\not p+m) /\left(p^{2}+m^{2}\right)^{1-\varepsilon}$, which mimics dimension $2+\varepsilon$, is certainly possible and was not included here only because of lack of energy. Even somewhat harder problems like the construction of the $\mathrm{GN}_{3}$ model at large $N$ or the dynamic mass generation in $\mathrm{GN}_{2}$ at large $N$ seem within reach of our methods.

During the preparation of this paper, we learned that Gawedzki and Kupiainen had obtained results which overlap strongly with ours [13]. 


\section{The Model}

\section{Notations}

In this paper any unimportant numerical constant will be called $O(1)$.

The massive two dimensional Euclidean $\mathrm{GN}_{2}$ model is a theory of $N$ component fermionic fields $(N>1)$, the lagrangian density being:

$$
L(\bar{\psi}, \psi)=\bar{\psi}(i \zeta \not \partial+m) \psi-\lambda(\bar{\psi} \cdot \psi)^{2}
$$

with $\bar{\psi} \cdot \psi=\sum_{a, \alpha} \bar{\psi}_{a}^{\alpha} \cdot \psi_{a}^{\alpha}$; the letters $a, b, \ldots$ will be used for spinor indices and take the value 0 or 1 ; the letters $\alpha, \alpha^{\prime}, \ldots$ will be used for fermionic indices and take the values $1, \ldots, N$. Since they are largely irrelevant to our analysis, we will forget them most of the time. In (2.1), we will perform a mass, a coupling constant and a wavefunction renormalization; as will be explained below, the wave function renormalization which shows up in perturbation theory will turn out to be finite. In (2.1) $m$ is the mass and the parameter $\zeta$ will be called the "wave-function" for short.

In the rest of the paper $\Lambda$ will denote a compact square box in $\mathbb{R}^{2}$, of area $|\Lambda|$, with integer side size, and some specified boundary conditions (which will be irrelevant). We pick a number $M>1$, and consider the sequence of energy scales 1 , $M, M^{2}, \ldots, M^{\circ}$; the parameter $\varrho$ is the index of the ultra-violet cutoff. We choose a cutoff of Pauli Villars type [so that we can easily factorize the propagator as in (2.8)],

$$
\begin{aligned}
& \eta_{\varrho}(p, \underline{m}, \underline{\zeta})
\end{aligned}
$$

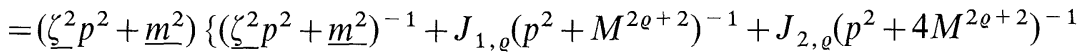

$$
\begin{aligned}
& \left.+J_{3, \varrho}\left(p^{2}+8 M^{2 \varrho+2}\right)^{-1}+J_{4, \varrho}\left(p^{2}+16 M^{2 \varrho+2}\right)^{-1}\right\} \\
& =J_{5, \varrho}\left(p^{2}+M^{2 \varrho+2}\right)^{-1}\left(p^{2}+4 M^{2 \varrho+2}\right)^{-1}\left(p^{2}+8 M^{2 \varrho+2}\right)^{-1} \\
& \cdot\left(p^{2}+16 M^{2 \varrho+2}\right)^{-1} \text {, }
\end{aligned}
$$

where the constants $J_{1}$ are uniquely determined by (2.2):

$$
\begin{aligned}
& J_{5, \varrho}=512 M^{8(\varrho+1)}\left(1-\underline{m}^{2} / M^{2 \varrho+2} \underline{\zeta^{2}}\right)\left(1-m^{2} / 4 M^{2 \varrho+2} \underline{\zeta^{2}}\right) \\
& \cdot\left(1-\underline{m^{2}} / 8 M^{2 \varrho+2} \underline{\zeta^{2}}\right)\left(1-\underline{m^{2}} / 16 M^{2 \varrho+2} \underline{\zeta^{2}}\right) \text {, } \\
& J_{1, \varrho}=-\left(\frac{512}{315 \underline{\zeta^{2}}}\right)\left(1-\underline{m^{2}} / 4 M^{2 \varrho}+2 \underline{\zeta^{2}}\right)\left(1-\underline{m^{2}} / 8 M^{2 \varrho+2} \underline{\zeta^{2}}\right) \\
& \cdot\left(1-\underline{m}^{2} / 16 M^{2 \varrho}+2 \underline{\zeta^{2}}\right) \text {, } \\
& J_{2, \varrho}=\left(\frac{8}{9 \underline{\zeta^{2}}}\right)\left(1-\underline{m^{2}} / M^{2 \varrho}+2 \underline{\zeta^{2}}\right)\left(1-\underline{m^{2}} / 8 M^{2 \varrho+2} \underline{\zeta^{2}}\right) \\
& \cdot\left(1-\underline{m^{2}} / 16 M^{2 \varrho}+2 \underline{\zeta^{2}}\right), \\
& J_{3, \varrho}=-\left(\frac{2}{7 \underline{\zeta^{2}}}\right)\left(1-\underline{m^{2}} / M^{2 \varrho}+2 \underline{\zeta^{2}}\right)\left(1-\underline{m^{2}} / 4 M^{2 \varrho}+2 \underline{\zeta^{2}}\right) \\
& \cdot\left(1-\underline{m^{2}} / 16 M^{2 \varrho}+2 \underline{\zeta^{2}}\right) \text {, } \\
& J_{4, \varrho}=\frac{1}{45 \underline{\zeta^{2}}}\left(1-\underline{m^{2}} / M^{2 \varrho} \underline{\underline{\zeta^{2}}}\right)\left(1-\underline{m^{2}} / 4 M^{2 \varrho}+2 \underline{\zeta^{2}}\right) \\
& \cdot\left(1-\underline{m^{2}} / 8 M^{2 \varrho+2} \underline{\zeta^{2}}\right) \text {. }
\end{aligned}
$$


The fermionic propagator with momentum cutoff $\varrho$ and bare mass and "wavefunction" $m_{\varrho}$ and $\zeta_{\varrho}$ is:

$$
C_{\varrho ; a, b}(x-y)=\int \eta_{\varrho}\left(p, m_{\varrho}, \zeta_{\varrho}\right) e^{i p \cdot(x-y)}\left(\zeta_{\varrho} p+m_{\varrho}\right)_{a, b} /\left(\zeta_{\varrho}^{2} p^{2}+m_{\varrho}^{2}\right) d^{2} p,
$$

where our conventions for $\gamma$ matrices are:

$$
\begin{gathered}
\gamma_{0}=\left(\begin{array}{cc}
i & 0 \\
0 & -i
\end{array}\right), \quad \gamma_{1}=\left(\begin{array}{cc}
0 & 1 \\
-1 & 0
\end{array}\right), \\
\not p=p_{0} \gamma_{0}+p_{1} \gamma_{1} ; \quad \gamma_{a}=-\gamma_{a}^{*} ; \quad\left\{\gamma_{a}, \gamma_{b}\right\}=-2 \delta_{a, b} .
\end{gathered}
$$

The normalized $2 p$-point Schwinger function (with cutoffs $\varrho$ and $\Lambda$ ) is:

$$
S_{2 \varrho, \Lambda, \varrho}\left(y_{1, c_{1}}, \ldots, y_{p, c_{p}} ; z_{1, d_{1}}, \ldots, z_{p, d_{p}}\right)=\mathbf{S}_{2 p, \Lambda, \varrho}(\{y\},\{z\}) / Z_{\Lambda, \varrho} .
$$

(These functions are in fact distributions and should be smeared against testfunctions.) The unnormalized Schwinger function $\mathbf{S}_{2 p, \Lambda, \varrho}$ has a perturbative expansion:

$$
\begin{gathered}
\mathbf{S}_{2 p, \Lambda, \varrho}(\{y\},\{z\})=\sum_{n=0, \ldots, \infty} \int_{\Lambda} d^{2} x_{1} \ldots d^{2} x_{n}\left[\left(\lambda_{\varrho}\right)^{n} / n !\right] \sum_{a_{i}, b_{i}} \ldots \\
\left\{\begin{array}{lllll}
y_{1, c_{1}} \ldots y_{p, c_{p}} & x_{1, a_{1}} & x_{1, b_{1}} \ldots x_{n, a_{n}} & x_{n, b_{n}} \\
z_{1, d_{1}} \ldots z_{p, d_{p}} & x_{1, a_{1}} & x_{1, b_{1}} \ldots x_{n, a_{n}} & x_{n, b_{n}}
\end{array}\right\},
\end{gathered}
$$

where $Z_{\Lambda, \varrho}$ is the normalization, $\lambda_{\varrho}$ is the bare coupling constant, the indices $a, b, c$, $d$ take the value 0 or 1 , and we use Cayley's notation for determinants

$$
\left(\begin{array}{l}
u_{i, a} \\
v_{j, b}
\end{array}\right)=\operatorname{det}\left(C_{\varrho ; a, b}\left(u_{i}-v_{j}\right)\right) .
$$

Also we decompose the fermion propagator in two parts:

$$
C_{\varrho ; a, b}(x-y)=\int A_{\varrho ; a, b}\left(x-t, m_{\varrho}, \zeta_{\varrho}\right) B_{\varrho}\left(t-y, m_{\varrho}, \zeta_{\varrho}\right) d^{2} t
$$

with

$$
A_{\varrho}(p, \underline{m}, \underline{\zeta})=(\underline{\zeta} \not p+\underline{m})\left(\underline{\zeta^{2}} p^{2}+\underline{m}^{2}\right)^{-1}\left(p^{2}+M^{2 \varrho+2}\right)^{-1}\left(p^{2}+16 M^{2 \varrho+2}\right)^{-1} M^{9 \varrho / 2},
$$

and

$$
B_{\varrho}(p m, \underline{\zeta})=J_{5, \varrho} M^{-9 \varrho / 2}\left(p^{2}+4 M^{2 \varrho+2}\right)^{-1}\left(p^{2}+8 M^{2 \varrho+2}\right)^{-1} .
$$

In the rest of the paper it is convenient to consider the $A$ and $B$ terms as "legs," or, with some abuse of language, as "fields." We remark indeed that a propagator is made of two "legs" and also the contraction of two fields $\psi$ and $\tilde{\psi}$.

\section{The Perturbative Renormalizability}

In this subsection we show that the theory is perturbatively renormalizable. It seems indeed that this has been checked only to lowest orders in [2].

Let us write the Schwinger functions as integrals over the anticommuting (Grassmann) field variables $\bar{\psi}$ and $\psi$ :

$$
\begin{gathered}
\mathbf{S}_{2 p, \Lambda, \varrho}(\{y\},\{z\})=\sum_{n}\left[\left(\lambda_{\varrho}\right)^{n} / n !\right] \int \psi_{c_{1}}\left(y_{1}\right) \ldots \psi_{c_{p}}\left(y_{p}\right) \bar{\psi}_{d_{p}}\left(z_{p}\right) \ldots \\
\bar{\psi}_{d_{1}}\left(z_{1}\right)\left\{\int_{\Lambda}(\bar{\psi} \cdot \psi)^{2}(x) d^{2} x\right\}^{n} d \mu_{\varrho}(\bar{\psi}, \psi)
\end{gathered}
$$


where, by definition of the gaussian measure $d \mu_{\varrho}$ :

$$
\int \psi_{a}(x) \cdot \bar{\psi}_{b}(y) d \mu_{\varrho}(\bar{\psi}, \psi)=C_{\varrho ; a, b}(x-y) \text {. }
$$

Lemma 2.1. The counterterms of the theory are of the form:

$$
\left\{\sum \bar{\psi}_{a} \cdot \psi_{a}\right\}^{2}, \quad \sum \bar{\psi}_{a} \cdot \psi_{a}, \quad \text { and } \sum \bar{\psi}_{a}\left(i \phi_{a, b}\right) \psi_{b}
$$

Proof. It is similar to Furry's theorem. By power counting the counterterms of the theory can only be quadratic or quartic in the fields $\bar{\psi}$ and $\psi$. We consider first the quartic case (coupling constant renormalization). In any graph we can define chains of fields: at a given vertex we will say that two fields are in the same chain if they belong both to the same scalar product $\sum \bar{\psi}_{a} \cdot \psi_{a}$ in the quartic term $\left\{\sum \bar{\psi}_{a} \cdot \psi_{a}\right\}^{2}$ at the vertex; we will also say that they are in the same chain if they are joined by a propagator.

If we consider a 4-point function, the external fields can obviously be divided into two pairs, each containing one field $\psi$ and one field $\bar{\psi}$, that are at opposite ends of a chain.

Now let us expand each propagator $(p+m) /\left(p^{2}+m\right)$ as the sum of $\left(p_{0} \gamma_{0}+m\right) /\left(p^{2}+m\right)$ and $p_{1} \gamma_{1} /\left(p^{2}+m\right)$; since $\gamma_{0}$ is diagonal and $\gamma_{1}$ is antidiagonal, the external fields of a chain have different indices $a$ and $b$ if and only if there is an odd number of $\gamma_{1}$ 's in the chain. Moreover, by parity considerations if the zero momentum value of the graph is non-zero, it contains an even number of $\gamma_{0}$ and $\gamma_{1}$ 's. By construction, apart from the two chains connecting the external fields, the diagram consists of closed chains, each of which contain an even number of $\gamma_{1}$ 's. Therefore, the only two possibilities for the two chains connecting the external fields are:

- An even number of $\gamma_{1}$ 's in each chain; the corresponding counterterms is then of the desired form $\left\{\sum \bar{\psi}_{a} \cdot \psi_{a}\right\}^{2}$;

- An odd number of $\gamma_{1}$ 's is on each chain; the corresponding counterterms are of the form $\bar{\psi}_{a} \psi_{b} \bar{\psi}_{a} \psi_{b}$ or $\bar{\psi}_{a} \psi_{b} \bar{\psi}_{b} \psi_{a}, a \neq b$. By permutation:

$$
\bar{\psi}_{a} \psi_{b} \bar{\psi}_{a} \psi_{b}=-\bar{\psi}_{a} \psi_{b} \bar{\psi}_{a} \psi_{b}=0, \quad \text { and } \quad \bar{\psi}_{a} \psi_{b} \bar{\psi}_{b} \psi_{a}=-\bar{\psi}_{a} \psi_{a} \bar{\psi}_{b} \psi_{b} .
$$

Thus in the first case the counterterm vanishes, and the second case, if we add the terms $\bar{\psi}_{a} \psi_{a} \bar{\psi}_{a} \psi_{a}$ which are zero by permutation, it can be reexpressed as $\sum_{a, b \neq a} \bar{\psi}_{a} \psi_{b} \bar{\psi}_{b} \psi_{a}=-\left\{\sum \bar{\psi}_{a} \cdot \psi_{a}\right\}^{2}$. Therefore, the quartic counterterms are of the form announced at least for the case of one colour. For the case of more than one colour, the divergent part of the terms which are not of the form $(\bar{\psi} \psi)^{2}$ cancel in pairs using appropriate combinations; this is because this divergent part is the same as for the massless theory of which the propagator is odd. It remains to prove the Lemma for the quadratic counterterms. But for the mass term, only the chain connecting the external fields can contain an odd number of $\gamma_{1}$ 's, and this possibility would give 0 by parity considerations on $p_{1}$. The remaining possibility is an even number of $\gamma_{1}$ 's in the chain, which gives a counterterm of the form $\sum \bar{\psi}_{a} \cdot \psi_{a}$. Finally for the wave-function term the argument is similar to the mass term and we leave it to the reader. 
The terms with four fermions which are not of type $(\bar{\psi} \psi)^{2}$ are convergent and will not be renormalized. The terms with two fermions which are not of type $\bar{\psi} \psi$ or $\bar{\psi} \phi \psi$ vanish at zero momenta and so are renormalized by construction.

\section{The Convergence of the Bare Unnormalized Series}

Gram's inequality tells us that:

$$
\left|\operatorname{det}\left(\int f_{i}(x) g_{j}(x) d x\right)\right| \leqq \prod_{i}\left\|f_{i}\right\|_{2} \cdot\left\|g_{i}\right\|_{2} .
$$

If we apply this inequality to the r.h.s. of (2.6) we obtain:

$$
\begin{gathered}
\left|\mathbf{S}_{2 p, \Lambda . \varrho}(\{y\},\{z\})\right| \leqq \sum_{n} \int_{A} d^{2} x_{1} \ldots d^{2} x_{n}\left[\left(\lambda_{\varrho}\right)^{n} / n !\right] \ldots \\
\left\{\left(\sum_{a}\left\|A_{\varrho ; a}\right\|_{2}\right)\left(\sum_{a}\left\|B_{\varrho ; a}\right\|_{2}\right)\right\}^{2 n} \cdot \prod_{i=1, \ldots, p}\left\|A_{\varrho ; c_{i}}\right\|_{2}\left\|B_{\varrho ; d_{i}}\right\|_{2},
\end{gathered}
$$

where

$$
\left\|A_{\varrho ; a}\right\|_{2}=\left\{\sum_{b} \int_{A} A_{\varrho ; a, b}(x) \bar{A}_{\varrho ; b, a}(x) d^{2} x\right\}^{1 / 2} \leqq O(1) M^{\varrho / 2} ;
$$

the same formula is true for $B$. Therefore:

$$
\left|\mathbf{S}_{2 p, \Lambda, \varrho}(\{y\},\{z\})\right| \leqq \sum_{n}\left[\left(\lambda_{\varrho}\right)^{n} / n !\right]\left[O(1) M^{\varrho}\right]^{p+2 n}|\Lambda|^{n},
$$

and we have proved:

Lemma 2.2. The unnormalized Schwinger functions $\mathbf{S}_{2 p, \Lambda, \varrho}(\{y\},\{z\})$, in a finite volume $\Lambda$ and with a finite ultra-violet cutoff $M^{\circ}$ are entire functions of the bare coupling $\lambda_{\varrho}$ (i.e., analytic in the whole complex plane), for any (finite!) value of the bare mass $m_{\varrho}$ and wave-function $\zeta_{\varrho}$.

Thanks to the Pauli principle, the radius of convergence of the expansion (2.6) is therefore infinite, in contrast with the case of bosonic theories like $\Phi^{4}$ in any dimension, where the radius of convergence of the unnormalized and unrenormalized Schwinger functions with cutoffs is zero.

\section{The Approximate Renormalization Group Flow}

We want to discuss in elementary terms the flow of the bare constants $\lambda_{\rho}, m_{e}$, and $\zeta_{\varrho}$ under a "renormalization group" transformation which integrates the momenta in the $\varrho^{\text {th }}$ slice. For the moment the reader may take it as a purely heuristic analysis, which will be justified rigorously by the next sections. By parity considerations, the mass renormalization is only logarithmic. Although there exist, at any order, infinite (logarithmically divergent) mass and wave function perturbative renormalizations (i.e., corresponding to divergent graphs of the two-point function), after asymptotic freedom is taken into account, only the mass renormalization generated by the "tadpole" (linear in the coupling constant) leads to non-trivial effects. Indeed any other two-point graph has at least 2 vertices, hence, by asymptotic freedom, has a "convergent logarithmic power counting" at large 
energy. We remark that planar $-g \Phi_{4}^{4}$ theory was more complicated in this respect since for any two-point graph the mass renormalization was quadratic and infinite even after renormalization group improvement.

Taking into account the most divergent graphs, the renormalization-group equations tell us that, at lowest orders in $\lambda_{\varrho}[1,2]$ :

$$
\begin{gathered}
\lambda_{\varrho-1} \simeq \lambda_{\varrho}\left\{1-(\log M)\left[\beta_{2}\left(\lambda_{\varrho} / \zeta_{\varrho}^{2}\right)+\left(-\beta_{2}^{2}(\log M)+\gamma_{3}\right)\left(\lambda_{\varrho} / \zeta_{\varrho}^{2}\right)^{2}\right]+O\left(\lambda_{\varrho}^{3}\right)\right\}, \\
m_{\varrho-1} \simeq m_{\varrho}\left\{1-\gamma(\log M)\left(\lambda_{\varrho} / \zeta_{\varrho}^{2}\right)+O\left(\lambda_{\varrho}^{2}\right)\right\}, \\
\zeta_{\varrho-1} \simeq \zeta_{\varrho}\left\{1+\gamma_{2}(\log M)\left(\lambda_{\varrho} / \zeta_{\varrho}^{2}\right)^{2}+O\left(\lambda_{\varrho}^{3}\right)\right\},
\end{gathered}
$$

where $\beta_{2}=-2(N-1) / \pi$ is negative, as is characteristic of an asymptotically free theory, and $\gamma=-(2 N-1) / \pi, \gamma_{2}=(2 N-1) /(2 \pi)^{2}[1,2]$; one defines $\beta_{3}=\gamma_{3}+2 \gamma_{2}$, $\beta_{3}=2(\mathrm{~N}-1) / \pi^{2}$ has been computed in [23], and so $\gamma_{3}=\left(N-\frac{3}{2}\right) / \pi^{2}$.

It is therefore elementary to compute the following asymptotic behavior of $\lambda_{\varrho}, m_{\varrho}$, and $\zeta_{\varrho}$ for large $\varrho$, starting from finite values $\lambda_{0}$ positive, $m_{0}$ and $\zeta_{0}$ :

$$
\begin{gathered}
\lambda_{\varrho} \simeq \zeta^{2}\left[-\beta_{2}(\log M) \varrho+\left(\beta_{3} / \beta_{2}\right) \log \varrho+C\right]^{-1} \equiv f(\varrho, C)^{-1}, \\
m_{\varrho} \simeq m \cdot \varrho^{-(N-1 / 2) /(N-1)}, \\
\zeta_{\varrho} \simeq \zeta,
\end{gathered}
$$

where $C, m$, and $\zeta$ are three constants, which parametrize the theory; the constant $m$ simply fixes the energy scale of the problem. (We may choose the system of units so that it has the value 1.)

Remark. Since there is an overall scale invariance $\left(\zeta \rightarrow 1, m \rightarrow m / \zeta, \lambda \rightarrow \lambda / \zeta^{2}\right)$, it is convenient, without any loss of generality, to choose $\zeta_{\varrho} \equiv \zeta=1$ in (2.22) and (2.1). We will always assume from now on that this choice has been made.

Our initial strategy [4] was to construct the $\mathrm{GN}_{2}$ model by summing the bare series, since this would allow a particularly clear verification of the axioms (see Sect. IV). This is unfortunately not possible in this simple form since the mass flow (2.21) gives a renormalized mass of order 1 only for positive $\lambda_{\varrho}$ chosen according to (2.20). We found after an elementary analysis that the ansatz (2.24) for $m_{\varrho}$ will always give a finite non-zero mass $m_{0}$, which is necessary for our last cluster expansion in Sect. III to converge. Therefore, it is not the usual bare series, but the bare series with mass renormalization which has a radius of convergence corresponding to asymptotic freedom, as announced in [4]. (Of course it corresponds to a bare theory, but with a $\lambda_{\varrho}$-dependent ansatz for $m_{\varrho}$, which is therefore more complicated than (2.21); one can check by an elementary analysis that this $\lambda_{\varrho}$-dependent ansatz is asymptotically of the form:

$$
m_{\varrho}=m \cdot\left\{1-\left[\beta_{2}(\log M)+f(\varrho, C)\right] \lambda_{\varrho}\right\}^{(N-1 / 2) /(N-1)} .
$$

We remark that for $\lambda_{\varrho}=f(\varrho, C)^{-1},(2.23)$ is asymptotic to (2.21).

Therefore, the final ansatz under which we will work is the following

$$
\begin{gathered}
m_{\varrho}=m \cdot\left\{1-\left[\beta_{2}(\log M)+f(\varrho, C)\right] \lambda_{\varrho}\right\}^{(N-1 / 2) /(N-1)}, \\
\zeta_{\varrho}=1 .
\end{gathered}
$$


Then we will prove that under the condition $\left|\lambda_{\varrho}\right| \leqq f(\varrho, C)^{-1}, C$ large enough, the perturbation series in $\lambda_{\varrho}$ converge absolutely (and uniformly in $\varrho$ ), and we will use this result to construct a two parameter family of theories, parametrized by $C$ and $m$, and relate it to the renormalized perturbation series in the usual BPHZ Scheme of subtractions at 0 external momenta, where the $\mathrm{BPHZ}$ renormalized parameters $\lambda_{\text {ren }}, m_{\text {ren }}$, and $\zeta_{\text {ren }}$ are complicated (but finite!) functions of $C$ and $m$.

\section{The Phase Space Expansion and the Thermodynamic Limit}

\section{The Cluster Expansion for the Theory with Fixed u.v. Cutoff}

In this subsection we will work at fixed u.v. cutoff $M^{e}$, hence under the simplest possible ansatz

$$
m_{\varrho} \text { fixed; } \quad \zeta_{\varrho}=1
$$

for the bare mass and wave function of the theory. We will prove that the bare expansion for the normalized functions $S_{2 p, e}$ with fixed ultraviolet cutoff, which are the limits of $S_{2 p, A, \varrho}$ as $\Lambda$ tends to $\mathbb{R}^{2}$, has still a non-zero radius of convergence (which depends of the ultraviolet cutoff $M^{o}$ ). One can obtain this result with a single cluster and Mayer expansion which we present now as a simple pedagogical example (we will perform in the next subsection a sequence of cluster expansions, one for each scale of momenta $1, M, \ldots, M^{e}$, as in [9] and call "phase space expansion" this more complicated expansion).

Let us consider the lattice $\mathbf{D}$ of the squares $\Delta$ in $\Lambda$, centered on $\mathbb{Z}^{2}$, and having unit side. The set of pairs of different squares $\left\{\Delta, \Delta^{\prime}\right\}, \Delta^{\prime} \neq \Delta$ of $\mathbf{D}$ is called $\mathbf{B}$. To each such pair $b$ we associate a link variable $s_{b}, 0 \leqq s_{b} \leqq 1$, which we use to expand the coupling between $\Delta$ and $\Delta^{\prime}$; but we introduce the $s_{b}$ dependence on the "legs" $A_{e}$ and $B_{e}$ of (2.8) in order to preserve the symmetric form of the determinant (2.7) and Gram's inequality. Hence we define:

$$
A_{\varrho}\left(\left\{s_{b}\right\}, x, y\right)=\left[\sum_{\Delta \in \mathbf{D}} \chi_{\Delta}(x) \chi_{\Delta}(y)+\sum_{b \in \mathbf{B}} s_{b} \chi_{b}(x, y)\right] A_{\varrho}(x, y)
$$

with $\chi_{b}(x, y)=\chi_{\Delta}(x) \chi_{\Delta^{\prime}}(y)+\chi_{\Delta^{\prime}}(x) \chi_{\Delta}(y)$.

We have $\left.A_{\varrho}(\{s\}, x, y)\right|_{s=1}=A_{\varrho}(x, y)$ and $\left|A_{\varrho}(\{s\}, x, y)\right| \leqq\left|A_{\varrho}(x, y)\right|$. We define also the obvious equivalents for the $B$ fields. The propagator $C_{\rho}(\{s\} x, y)$ is defined by replacing $A_{\varrho}$ and $B_{\varrho}$ by $A_{\varrho}(\{s\})$ and $B_{\varrho}(\{s\})$ in (2.8), and $\mathbf{S}_{2 p, A, \varrho}(\{s\})$ is defined by replacing $C_{\rho}(x, y)$ by $C_{\rho}(\{s\} x, y)$ in (2.6)-(2.7). Also the gaussian measure corresponding to the propagator $C_{\varrho}(\{s\} x, y)$ is called $d \mu_{\rho}(\{s\})$.

The cluster expansion that we use is similar to the one used in [8]; slightly simpler than the "tree" expansion of Brydges, Battle, and Federbush (for a review see [14]) but not as powerful, it is nevertheless sufficient for theories with exponential decay of correlations as here. We will try to be both self-contained and reasonably brief. To compute $\mathbf{S}_{2 p, \Lambda, \varrho}$ we apply inductively, for each "bond" $b$, a Taylor formula to first order in $s_{b}$ to $\mathbf{S}_{2 p, A, \varrho}(\{s\})$. More precisely let $I_{b}$ and $J_{b}$ be defined by:

$$
I_{b} F(\{s\})=F(\{s\})_{s_{b}=0} ; \quad J_{b} F(\{s\})=\int_{0}^{1}\left(d F(\{s\}) / d s_{b}\right) d s_{b} .
$$


Then the cluster expansion may be written symbolically as:

$$
\mathbf{S}_{2 p, \Lambda, \varrho}=\left.\mathbf{S}_{2 p, \Lambda, \varrho}\right|_{s_{b}=1 \forall b \in \mathbf{B}}=\prod_{b \in \mathbf{B}}\left(I_{b}+J_{b}\right) \mathbf{S}_{2 p, \Lambda, \varrho}(\{s\}) .
$$

Let us compute:

$$
\begin{aligned}
& \left(d / d s_{b}\right) \operatorname{det}\left(\int A_{\varrho}\left(\{s\}, u_{i}-t\right) B_{\varrho}\left(\{s\}, t-v_{j}\right) d t\right) \\
& =\sum_{i_{0}, j_{0}}(-1)^{i_{0}+j_{0}} \operatorname{det}\left(\left.\int A_{\varrho}\left(\{s\}, u_{i}-t\right) B_{\varrho}\left(\{s\}, t-v_{j}\right) d t\right|_{i \neq i_{0}, j \neq j_{0}}\right) \\
& \quad \cdot\left(d / d s_{b}\right)\left[\int A_{\varrho}\left(\{s\}, u_{i_{0}}-t\right) B_{\varrho}\left(\{s\}, t-v_{j_{0}}\right) d t\right],
\end{aligned}
$$

or with the field notation:

$$
\begin{aligned}
\left(d / d s_{b}\right) \int \prod_{i} \bar{\psi}\left(u_{i}\right) \prod_{j} \psi\left(v_{j}\right) d \mu_{\varrho}(\{s\}) & \\
= & -\int\left(d C_{\varrho}(\{s\} x, y) / d s_{b}\right) \delta / \delta \psi(x) \delta / \delta \bar{\psi}(y) \\
& \cdot \prod_{i} \bar{\psi}\left(u_{i}\right) \prod_{j} \psi\left(v_{j}\right) d \mu_{\varrho}(\{s\}) d x d y .
\end{aligned}
$$

We remark that since $A_{\varrho}(\{s\})$ and $B_{\varrho}(\{s\})$ are linear in each variable $s_{b}$, a given propagator can be derived at most twice. Now we expand (3.4):

$$
\prod_{b \in \mathbf{B}}\left(I_{b}+J_{b}\right) \mathbf{S}_{2 p, \Lambda, \varrho}(\{s\})=\sum_{H \subset \mathbf{B}}\left(\prod_{b \notin H} I_{b}\right)\left(\prod_{b \in H} J_{b}\right) \mathbf{S}_{2 \dot{p}, \Lambda, \varrho}(\{s\}),
$$

and we say that two squares $\Delta$ and $\Delta^{\prime}$ of $\mathbf{D}$ are connected if there exists a link from $\Delta$ to $\Delta^{\prime}$ through bonds of $H$. (Remark that in contrast with the "tree" cluster [14] there might be loops in $H$.)

A set $\Gamma$ of one or more squares of $\mathbf{D}$ will be called a "vacuum polymer" $(\emptyset$ polymer) (see $[14,15])$. Let us define $\mathbf{B}_{c}(\Gamma)$ as the set of those subsets $H$ of $\mathbf{B}$ such that each bond in $H$ joins two squares in $\Gamma$ and altogether they connect all squares of $\Gamma$. Using the field notation as in (3.6) we define the "activity" $K(\Gamma)$ of a $\emptyset$-polymer $\Gamma$ :

$$
K(\Gamma)=\int \sum_{H \in \mathbf{B}_{c}(\Gamma)}\left(\prod_{b \notin H} I_{b}\right)\left(\prod_{b \in H} J_{b}\right)_{n=1, \ldots, \infty}\left(\lambda_{\varrho}\right)^{n} / n !\left\{\int_{\Gamma}(\bar{\psi} \cdot \psi)^{2}\right\}^{n} d \mu_{\varrho}(\{s\})
$$

or, with the determinant notation of (2.6):

$$
\begin{aligned}
K(\Gamma)= & \sum_{n=1, \ldots, \infty}\left(\lambda_{\varrho}\right)^{n} / n ! \int_{\Gamma} d^{2} x_{1} \ldots d^{2} x_{n} \sum_{H \in \mathbf{B}_{c}(\Gamma)}\left(\prod_{b \notin H} I_{b}\right)\left(\prod_{b \in H} J_{b}\right) \\
& \cdot \sum_{a_{i}, b_{i}}\left(\begin{array}{lll}
x_{1, a_{1}} & x_{1, b_{1}} \ldots x_{n, a_{n}} & x_{n, b_{n}} \\
x_{1, a_{1}} & x_{1, b 1} \ldots x_{n, a n} & x_{n, b_{n}}
\end{array}\right)(s)
\end{aligned}
$$

with

$$
\left(\begin{array}{l}
u_{i, a} \\
v_{j, b}
\end{array}\right)(s)=\operatorname{det}\left(C_{\varrho ; a, b}\left(\{s\}, u_{i}-v_{j}\right)\right) .
$$

The sums in (3.8)-(3.9) start at $n=1$ since we do not want to consider "empty" polymers reduced to a single square without fields, whose activity would be 1 . if $\Gamma^{\prime}$ is a set of squares of $\mathbf{D}$ plus some (possibly not all) of the external variables $y_{i}, z_{j}$ 
localized in these squares, we call it an external polymer (e-polymer) and define its $K\left(\Gamma^{\prime}\right)$, by introducing in (3.8) the fields corresponding to those external variables (starting now the sum over $n$ at $n=0$ ). One has by a standard counting argument

$$
\left\{\int_{\Lambda}\left(\lambda_{\varrho}\right)(\bar{\psi} \cdot \psi)^{2}\right\}^{n} / n !=\sum_{\left\{n_{\Gamma} \mid \sum_{\Gamma} n_{\Gamma}=n\right\}} \prod_{\Gamma}\left[\left\{\int_{\Gamma}\left(\lambda_{\varrho}\right)(\bar{\psi} \cdot \psi)^{2}\right\}^{n_{\Gamma}} / n_{\Gamma} !\right]
$$

for any family of disjoint polymers with $\bigcup_{\Gamma} \Gamma=\Lambda$, and therefore using (2.6), (3.4)-(3.7), and (3.9):

$$
\mathbf{S}_{2 p, A, \varrho}(\{y\},\{z\})=\sum_{\Gamma^{D}\{y, z\}} \mathbf{O} \prod_{\Gamma \in \Gamma^{D}\{y, z\}} K(\Gamma),
$$

where the sum is performed over the set $\Gamma^{D}\{y, z\}$ of all sets made of disjoint polymers (vacuum or external) such that any external variable $y$ or $z$ is contained in exactly one of them. (Two polymers, vacuum or external, are called disjoint if they have no common squares and are called "overlapping" otherwise.) The symbol $\mathbf{O}$ stands for the sign required to permute the external variables of the $K(\Gamma)$ 's to their order in $\mathbf{S}_{2 p, \Gamma, \varrho}(\{y\},\{z\})$.

We are ready to perform now a Mayer expansion, i.e., to rewrite (3.11) in a suitable form to perform the division by the normalization $Z_{\Gamma, \varrho}$ and to compute the normalized Schwinger functions $S_{2 p, \varrho}=\lim _{\Gamma \rightarrow \mathbb{R}^{2}} \mathbf{S}_{2 p, \Gamma, \varrho} / Z_{\Gamma, \varrho}$. First we introduce the notion of a "Mayer graph." It is a finite (ordered) sequence $\mathbf{M}=\left(\Gamma_{1}, \ldots, \Gamma_{p}\right)$ of polymers $\left(\emptyset-\right.$ or $\left.e^{-}\right)$which is linear in the external variables (i.e., for any such variable $y$ there is at most one polymer $\Gamma_{i}$ in the sequence $\mathbf{M}$ which contains $y$ ). We may index a Mayer graph by the set of all external variables present in its polymers and speak of a $\emptyset$-Mayer graph, of a $(\{y\},\{z\})$-Mayer graph, etc.... The integer $p=p(\mathbf{M})$ is the length of the Mayer graph. It is also useful to introduce the set $C(\mathbf{M})$ of all pairs $(i, j) 1 \leqq i<j \leqq p(\mathbf{M})$. The Mayer graph is called disjoint (respectively connected) if, for $1 \leqq i<j \leqq p, \Gamma_{i} \cap \Gamma_{j}=\emptyset$ (respectively if, for $1 \leqq i<j \leqq p$, there is a chain of "overlapping" polymers in the Mayer graph, $\left(\Gamma_{i}=\Gamma_{i_{1}}, \Gamma_{i_{2}}\right),\left(\Gamma_{i_{2}}, \Gamma_{i_{3}}\right), \ldots$, $\left(\Gamma_{i_{k-1}}, \Gamma_{i_{k}}=\Gamma_{j}\right)$, joining $\Gamma_{i}$ to $\left.\Gamma_{j}\right)$. The "amplitude of the Mayer graph is defined as:

$$
K(\mathbf{M})=\left[1 / p ! \prod_{i=1, \ldots, p} K\left(\Gamma_{i}\right)\right]
$$

Then we may write in (3.11) the constraints $\Gamma \cap \Gamma^{\prime}=\emptyset$, for two polymers $\Gamma$ and $\Gamma^{\prime}$ in $\Gamma\{y, z\}$ in terms of a two-body repulsive potential $V$, defined by $V\left(\Gamma, \Gamma^{\prime}\right)=0$ if $\Gamma$ and $\Gamma^{\prime}$ are disjoint and $V\left(\Gamma, \Gamma^{\prime}\right)=+\infty$ if $\Gamma$ and $\Gamma^{\prime}$ are overlapping. Therefore (3.11) may be written (since all polymers in a disjoint Mayer graph are distinct):

$$
\begin{aligned}
\mathbf{S}_{2 p, A, \varrho}(\{y\},\{z\}) & =\sum_{\operatorname{disjoint}(\{y\},\{z\})-\text { Mayer graphs } \mathbf{M}} \mathbf{O} K(\mathbf{M}) \\
& =\sum_{(\{y\},\{z\})-\text { Mayer graphs } \mathbf{M}} \mathbf{O} K(\mathbf{M}) \prod_{(i, j) \in C(\mathbf{M})} e^{-V\left(\Gamma_{i}, \Gamma_{j}\right)},
\end{aligned}
$$

since the product in (3.13) is 1 if $\mathbf{M}$ is disjoint and 0 otherwise. The Mayer expansion consists in writing $e^{-V\left(\Gamma_{i}, \Gamma_{j}\right)}=\left(e^{-V\left(\Gamma_{i}, \Gamma_{j}\right)}-1\right)+1$ and in expanding

$$
\prod_{(i, j) \in C(\mathbf{M})} e^{-V\left(\Gamma_{i}, \Gamma_{j}\right)}=\sum_{J C C(\mathbf{M})} \prod_{(i, j) \in J}\left[e^{-V\left(\Gamma_{i}, \Gamma_{j}\right)}-1\right] .
$$


The result of this expansion is well known $[14,15]$. A subset $J$ in (3.14) defines subsequences of $\mathbf{M}$ which are the maximal ones that are connected by the "bonds" of $J$. These subsequences may again be considered as Mayer graphs but, in contrast with $\mathbf{M}$, they are connected Mayer graphs [otherwise the corresponding contributions in (3.14) vanish]. The (truncated) "amplitude" $K^{T}(\mathbf{M})$ of a connected Mayer graph $\mathbf{M}$ is simply $T(\mathbf{M}) \cdot K(\mathbf{M})$, where $T(\mathbf{M})$ is an explicit combinatoric factor:

$$
T(\mathbf{M})=\sum_{J \in J^{T}(\mathbf{M})} \prod_{(i, j) \in J}\left[e^{-V\left(\Gamma_{i}, \Gamma_{j}\right)}-1\right]
$$

$J^{T}(\mathbf{M})$ being the set of all subsets of $C(\mathbf{M})$ connecting $\mathbf{M}$ into a single component [14]. Therefore, the result of the Mayer expansion may be expressed as a sum over "super-Mayer graphs" which are finite sequences of connected Mayer graphs and which, again, are linear in the external variables (i.e., each such variable appears at most once in one single Mayer graph of the sequence). Super-Mayer graphs may therefore also be indexed by their external variables. The "amplitude" $K(\mathbf{S})$ of a super-Mayer graph $\mathbf{S}=\left(\mathbf{M}_{1}, \ldots, \mathbf{M}_{r}\right)$ is simply $\left[1 / r ! \prod_{k=1, \ldots, r} K^{T}\left(\mathbf{M}_{k}\right)\right]$ and (3.13)
takes the compact form:

$$
\mathbf{S}_{2 p, \Lambda, \varrho}(\{y\},\{z\})=\sum_{(\{y\},\{z\}) \text {-super-Mayer graphs } \mathbf{S}} \mathbf{O} K(\mathbf{S}) .
$$

Although this is not obvious at first sight the normalization $Z_{\Gamma, \varrho}$ is directly factorized in (3.16). Indeed super-Mayer graphs may be reordered to put, in the sequence, their external Mayer graphs first, and then their $\emptyset$-May graphs. With a little trivial use of multinomial coefficients, (3.16) becomes:

$$
\begin{gathered}
\mathbf{S}_{2 p, A, \varrho}(\{y\},\{z\})=\sum_{\Omega=\left\{\omega_{1}, \ldots, \omega_{m}\right\}} \mathbf{O} \prod_{i=1, \ldots, m}\left\{\sum_{\mathbf{M} \omega_{1} \text {-Mayergraph }} K^{T}(\mathbf{M})\right\} \\
\sum_{n=0, \ldots, \infty} 1 / n !\left\{\sum_{\mathbf{M} \emptyset_{\text {-Mayer graph }}} K^{T}(\mathbf{M})\right\}^{n},
\end{gathered}
$$

where the sum is taken over partitions $\Omega$ of all external variables into $p$ subsets $\omega_{1}, \ldots, \omega_{m}$. In $(3.17)$

$$
Z_{\Lambda, \varrho}=\sum_{n=0, \ldots, \infty} 1 / n !\left\{\sum_{\mathbf{M} \emptyset_{\text {-Mayer graph }}} K^{T}(\mathbf{M})\right\}^{n}
$$

is factorized, and therefore:

$$
S_{2 p, \Lambda, \varrho}(\{y\},\{z\})=\sum_{\Omega=\left\{\omega_{1}, \ldots, \omega_{m}\right\}} \mathbf{O} \prod_{i=1, \ldots, m}\left\{\sum_{\mathbf{M} \omega_{l} \text {-Mayer graph }} K^{T}(\mathbf{M})\right\} .
$$

Moreover, one can easily verify that the usual truncated (normalized) Schwinger functions are just given by the case, where the partition $\Omega$ in (3.18) is trivial:

$$
S_{2 p, A, \varrho}^{T}(\{y\},\{z\})=\sum_{\mathbf{M}(\{y\},\{z\})-\text { Mayer graph }} K^{T}(\mathbf{M}) .
$$

Lemma 3.1. The expansions (3.18)-(3.19), for fixed bare mass $m_{\varrho}$, as power series in $\lambda_{\varrho}$ have a non-zero radius of convergence $r_{\varrho}\left(\Lambda, m_{\varrho}\right)$, which satisfies:

$$
\left[r_{\varrho}\left(\Lambda, m_{\varrho}\right)\right]^{-1} \leqq K\left(m_{\varrho}\right) M^{10 \varrho}=C_{\varrho} .
$$


This bound is uniform in $\Lambda$. The thermodynamic limits of $S$ and $S^{T}$ exist, uniformly in $\lambda_{\varrho}$ for $\left|\lambda_{\varrho}\right| \leqq\left(C_{\varrho}\right)^{-1}$ :

$$
\begin{aligned}
& S_{2 p, \varrho}(\{y\},\{z\})=\lim _{\Lambda \rightarrow \infty} S_{2 p, \Lambda, \varrho}(\{y\},\{z\}), \\
& S_{2 p, \varrho}^{T}(\{y\},\{z\})=\lim _{\Lambda \rightarrow \infty} S_{2 p, \Lambda, \varrho}^{T}(\{y\},\{z\}) .
\end{aligned}
$$

Therefore as power series in $\lambda_{\varrho}$ these normalized functions have a nonzero radius of convergence $r_{\varrho}\left(m_{\varrho}\right)$, satisfying $r_{\varrho}\left(m_{\varrho}\right) \geqq\left(C_{\varrho}\right)^{-1}$.

Remark. The $\varrho$-dependence of the bound (3.20) on $r_{\varrho}^{-1}$ is in fact very sloppy and will be greatly improved in Theorem IV.1 below, using the phase space analysis of the next sections [and choosing $m_{\varrho}$ according to (2.24)]. In what follows, $K$ will be used for a $m_{\varrho}$-dependent constant.

Proof. Let $|\Gamma|$ be the number of cubes of the lattice $\mathbf{D}$ in a polymer $\Gamma$. By the convergence criterium of [14] (which uses the Battle-Federbush theorem on treeprobability measures, the translation invariance of $K(\Gamma)$ and Cayley's theorem on the number of trees with given coordination numbers), the lemma follows from these two statements:

i) $\quad K(\Gamma)$ is analytic in $\lambda_{\varrho}$ (in the whole complex plane).

ii) For $\left|\lambda_{\varrho}\right| \leqq K \cdot M^{-10 \varrho}$, one has $\sum_{\Gamma \ni 0}|K(\Gamma)| e^{|\Gamma|}<1$.

Remark. This convergence criterion is rather sharp. With easy combinatoric arguments which do not use Battle-Federbush theorem, one could replace condition (3.24) by a weaker one, still satisfied in our case, where the theory is massive and the correlation decay exponentially at infinity.

Let us prove (3.23)-(3.24) for $\Gamma \emptyset$-polymer, the proof for $\Gamma$ external polymer being similar. From the explicit formula for $A^{\varrho}$ and $B^{\varrho}$ one gets easily:

$$
\sup \left\{\left|A_{\varrho}(\{s\}, x, y)\right|,\left|B_{\varrho}(\{s\}, x, y)\right|\right\} \leqq O(1) M^{3 \varrho / 2} e^{-m_{\varrho}|x-y| / 2},
$$

Let us prove first (3.23). We call $\Delta_{1}, \ldots, \Delta_{q}$ the squares of $\Gamma$. In (3.9), for $n>q(q-1) / 4$ we can bound the sum over $H \in \mathbf{B}_{c}(\Gamma)$ by a factor $2^{q(q-1) / 2}$ and, by (3.5) and (3.25), the action of the derivations $d / d s_{b}$ for $b \in H$ by $O(1)^{q(q-1) / 2}(2 n)^{q(q-1)} M^{3 \varrho[q(q-1)] / 2}$ times a reduced determinant, at most $2 n$ by $2 n$, which can be evaluated by Gram's inequality as in Sect.2.3. The factor $1 / n$ ! in (3.9) is therefore preserved, and the analyticity of $K(\Gamma)$ and $K_{e}(\Gamma)$ follows.

To prove (3.24) let us bound the "choice" of $\Gamma=\left\{\Delta_{1}, \ldots, \Delta_{q}\right\}$, such that $0 \in \Gamma$, using the decrease of $A$ and $B$ at large distance (3.25) due to the bare mass of the theory. For any fixed $H \in \mathbf{B}_{c}(\Gamma)$ we write (recall from now on that the sum is over $\emptyset-\Gamma$ 's):

$$
\begin{gathered}
\sum_{\Gamma \ni 0}|K(\Gamma)| e^{|\Gamma|}=\sum_{q} e^{q} \sum_{\Gamma \ni 0} \sum_{n_{1}, \ldots, n_{q}} \sum_{H \in \mathbf{B}_{c}(\Gamma)}, \\
\mid \prod_{i=1, \ldots, q}\left\{\left(\lambda_{\varrho}\right)^{n_{\imath}} / n_{i} ! \int_{\Delta_{\imath}} d^{2} x_{1}^{i} \ldots d^{2} x_{n_{i}}^{i}\right\}\left(\prod_{b \notin H} I_{b}\right)\left(\prod_{b \in H} J_{b}\right) \\
\cdot \sum_{a, b}\left(\begin{array}{lll}
x_{1, a_{1}}^{1} & x_{1, b_{1}}^{1} \ldots x_{n_{q}, a_{n}}^{q} & x_{n_{q}, b_{n}}^{q} \\
x_{1, a_{1}}^{1} & x_{1, b_{1}}^{1} \ldots x_{n_{q}, a_{n}}^{q} & x_{n_{q}, b_{n}}^{q}
\end{array}\right)(s)
\end{gathered}
$$


with $n=\sum_{i=1, \ldots, q} n_{i}$. Keeping $\Delta_{1}, \ldots, \Delta_{q}$ and $H$ fixed, we compute the action of the derivations on the determinant by (3.5). We can choose (paying with a factor $2^{|H|}$ ) whether a given $d / d s_{b}, b=\left\{\Delta_{i}, \Delta_{j}\right\}$, in (3.24) derives a leg $A\left(x_{p_{i}}^{i}, t\right)\left[\right.$ or $\left.B\left(t, x_{p_{i}}^{i}\right)\right]$ or derives a let $A\left(x_{p_{j}}^{j}, t\right)\left[\right.$ or $\left.B\left(t, x_{p_{j}}^{j}\right)\right]$, with $t \in \Delta_{j}$ in the first case and $t \in \Delta_{i}$ in the last case. For each given $\Delta_{i}$ we call $Q_{i}$ (respectively $S_{i}$ ) the set of different derivations $d / d s_{b}, b=\left\{\Delta_{i}, \Delta_{j}\right\}$, of the first type (respectively of the second type); it has $q_{i}=\left|Q_{i}\right|$ elements (respectively $s_{i}=\left|S_{i}\right|$ elements), with $\sum_{i} q_{i}=\sum_{i} s_{i}=|H|$. The sum over $H \in \mathbf{B}_{C}\left(\Delta_{1}, \ldots, \Delta_{q}\right)$ can be replaced now by a sum over the $Q_{i}$ 's, such that $U\left\{Q_{i}\right\} \in \mathbf{B}_{C}\left(\Delta_{1}, \ldots, \Delta_{q}\right)$. To compute the derivations $d / d s_{b}$ for $b \in Q_{i}$ we can choose with a factor $2^{4 n_{2}} \cdot q_{i}$ ! which legs of the $n_{i}$ vertices in $\Delta_{i}$ are associated to which derivation (since there are 4 legs per vertex). These legs are called "derived" legs. Then we have to choose the "underived" legs (joining $t \in \Delta_{j}$ to $x_{p_{k}}^{k} \in \Delta_{k}$ ) which complete "derived" legs into full propagators. Let us call $r_{k}^{j}$ the number of such "underived" legs and define $r_{k}=\sum_{j} r_{k}^{j}$. Paying with a factor $2^{4 n_{k}}$ we can choose $r_{k}$ and the total set of these $r_{k}$ "underived" legs. Paying with a factor $r_{k} ! / \prod_{j} r_{k}^{j}$ ! we choose which of these $r_{k}$ legs belong to each $r_{k}^{j}$. We have also to "pair together" in a square $\Delta_{j}$ the $s_{j}$ "derived legs" of the second type with the $t_{j}=\sum_{k} r_{k}^{j}$ "underived legs." Derived legs can be paired together (this corresponds to differentiating a propagator twice) but underived legs cannot, hence $s_{j}-t_{j}$ is positive and even, and the number of possible such pairings is bounded by $2^{s_{j}} s_{j}$ !. Finally, we bound the remaining determinant by Gram's inequality, as in (2.15). We obtain the following bound, which uses (3.25):

$$
\begin{aligned}
& \sum_{\Gamma \ni 0}|K(\Gamma)| e^{|\Gamma|} \leqq \sum_{q} e^{q} \sum_{\Gamma \ni 0} \sum_{n_{1}, \ldots, n_{q} / \Sigma n_{l}=n} O(1)^{n} \\
& \sum_{Q_{l} / \cup\left\{Q_{i}\right\} \in \mathbf{B}_{C}\left(\Delta_{1}, \ldots, \Delta_{q}\right)} \prod_{i=1, \ldots, q}\left\{\left(\lambda_{\varrho}\right)^{n_{z}} / n_{i} ! \int_{\Delta_{\imath}} d^{2} x_{1}^{i} \ldots d^{2} x_{n_{i}}^{i}\right\} \\
& \cdot \sup _{r_{l}=1, \ldots, n_{i}}\left\{\left[\prod_{i=1, \ldots, q} s_{i} ! q_{i} !\right]\left[O(1) M^{3 \varrho}\right]^{|H|}\left[\prod_{b \in H} e^{-\delta(b)}\right]\right. \\
& \cdot \sum_{r_{k}^{J} / \Sigma_{j} r_{k}^{j}=r_{k}}\left\{r_{k} ! \prod_{j}\left[e^{\left.-\delta(k, j) r_{k}^{j} /\left(r_{k}^{j}\right) !\right]}\right\}\left[O(1) M^{\varrho}\right]^{2 n-|H|}\right\},
\end{aligned}
$$

where, for $b=\left[\Delta_{i}, \Delta_{j}\right\}, \delta(b)=\delta(i, j)$ is defined as $\inf _{x \in \Delta_{i}, y \in \Delta_{j}}\left\{m_{\varrho} \cdot d(x, y) / 2\right\}$. To simplify this expression, we use first the multinomial theorem and the fact that $\sum_{j=1, \ldots, q} e^{-\delta(j, k)}$ is uniformly bounded by $K$. Then, since all bonds $b$ in $Q_{i}$ (respectively in $S_{i}$ ) are different, half of them are at least at a distance $O(1) q_{i}^{1 / 2}$ (respectively $\left.O(1) s_{i}^{1 / 2}\right]$ of $\Delta_{i}$, and therefore it is easy to prove that $s_{i} ! q_{i} ! \prod_{b \in Q_{i} \cup S_{i}} e^{-\delta(b) / 3} \leqq K^{q_{i}+s_{i}}$.

The integrations are over unit cubes. The sum over $n_{1}, \ldots, n_{q}$ such that $\sum n_{i}=n$ is bounded by $2^{n+q}$. Since any derivation $d / d s_{b}$ for $b \in H$ applies to at least one leg $A$ or $B$, since these legs are derived at most once, and since there are 4 half 
propagators per vertex, we see that there are at least $|H| / 4$ vertices in $\Gamma$; since $n \geqq 1$ in (3.9), we have in fact $n \geqq \sup \{1,|H| / 4\}$. Therefore, we obtain:

$$
\begin{aligned}
\sum_{\Gamma \ni 0}|K(\Gamma)| e^{|\Gamma|} \leqq & \sum_{q}(2 e)^{q} \sum_{\Gamma \ni 0} \sum_{n \geqq \sup \{1,|H| / 4\}}\left(O(1) M^{2 \varrho \varrho} \lambda_{\varrho}\right)^{n} \\
& \cdot \sum_{G_{i} / \cup\left\{Q_{i}\right\} \in \mathbf{B}_{c}\left(\Delta_{1}, \ldots, \Delta_{q}\right)} \cdot\left\{\prod_{i=1, \ldots, q}\left\{\left[K M^{2 \varrho}\right]^{q_{i}} \prod_{b \in Q_{i}} e^{-2 \delta(b) / 3}\right\}\right\} .
\end{aligned}
$$

We have for any $i \in[1, q]$ :

$$
\sum_{Q^{i}}\left\{\left[K M^{2 \varrho}\right]^{q_{i}} \prod_{b \in Q_{i}} e^{-\delta(b) / 3}\right\} \leqq\left[K M^{2 \varrho}\left(\sum_{j=1, \ldots, q} e^{-\delta(i, j) / 3}\right)\right]^{q_{i}} \leqq\left[K M^{2 \varrho}\right]^{q_{i}} .
$$

Moreover, for any $H \in \mathbf{B}_{C}\left(\Delta_{1}, \ldots, \Delta_{q}\right)$ there exists a permutation $\sigma$ of $[1, q]$ such that

$$
\prod_{b \in H} e^{-\delta(b) / 3} \leqq K^{|H|} e^{-\Sigma_{i}^{\delta}[\sigma(i), \sigma(i+1)] / 6} \leqq K^{|H|} \sum_{\sigma} e^{-\Sigma_{i} \delta[\sigma(i), \sigma(i+1)] / 6} .
$$

(Hint: pick a tree of $H$ and "turn around the tree" using triangular inequality as in [16].) Combining this fact with (3.29) we get from (3.28):

$$
\begin{aligned}
\sum_{\Gamma \ni 0}|K(\Gamma)| e^{|\Gamma|} \leqq & \sum_{q}(2 e)^{q} \sum_{\Gamma \ni 0} \sum_{n \geqq \sup \{1,|H| / 4\}} \\
& \cdot\left(O(1) M^{2 \varrho} \lambda_{\varrho}\right)^{n}\left[K M^{2 \varrho}\right]^{|H|} \sum_{\sigma} e^{-\Sigma_{i} \delta[\sigma(i), \sigma(i+1)] / 6} .
\end{aligned}
$$

Finally, since $\Delta_{1}, \ldots, \Delta_{q}$ are distinct, we can bound the sum over $\Gamma$ by $1 /(q-1)$ ! times the independent sum over $\Delta_{2}, \ldots, \Delta_{q}$ with $0 \in \Delta_{1}$ fixing $\Delta_{1}$. This sum is controlled by the exponential decay of (3.30), the total number $q$ ! of permutations $\sigma$ being controlled by the factor $1 /(q-1)$ !. Since $q \leqq|H|+1$ and $|H| \leqq 4 n$, we have finally:

$$
\sum_{\Gamma \ni 0}|K(\Gamma)| e^{|\Gamma|} \leqq \sum_{n \geqq 1}\left(K M^{10 \varrho} \lambda_{\varrho}\right)^{n},
$$

which achieves the proof of (3.24), hence of Lemma 3.1, if:

$$
\lambda_{\varrho}<(2 K)^{-1} M^{-10 \varrho} \text {. }
$$

\section{The Phase-Space Expansion}

We introduce momentum slices, one for each scale of momenta $1, M, \ldots, M^{k}, \ldots, M^{\varrho}$, as in [9]. For short, we identify a momentum slice with its "index" $k$. More precisely we write: $\eta_{\varrho}\left(p, m_{\varrho}, \zeta_{\varrho}\right)=\sum_{k=0, \ldots, \varrho} \eta^{k}\left(p, m_{\varrho}, \zeta_{\varrho}\right)$ with $\eta^{0}(p)=\eta_{0}(p)$ and for $k \geqq 1$ :

$$
\begin{aligned}
\eta^{k}(p, \underline{m}, \underline{\zeta})= & \eta_{k}(p, \underline{m}, \underline{\zeta})-\eta_{k-1}(p, \underline{m}, \underline{\zeta}) \\
= & \left(\underline{\zeta}^{2} p^{2}+\underline{m}^{2}\right) Q^{k}(p, \underline{m}, \underline{\zeta})\left[\left(p^{2}+16 M^{2 k+2}\right)\left(p^{2}+16 M^{2 k}\right)\right]^{-1} \\
& \cdot\left[\left(p^{2}+M^{2 k+2}\right)\left(p^{2}+4 M^{2 k+2}\right)\left(p^{2}+8 M^{2 k+2}\right)\right. \\
& \left.\cdot\left(p^{2}+M^{2 k}\right) \cdot\left(p^{2}+4 M^{2 k}\right)\left(p^{2}+8 M^{2 k}\right)\right]^{-1},
\end{aligned}
$$


where this defines $Q^{k}(p, \underline{m}, \underline{\zeta})$ which is a polynomial of sixth order in $p$. Also we define

$$
C_{\varrho, a, b}(x-y)=\sum_{k=0, \ldots, \varrho} C_{a, b}^{k}(x-y),
$$

with $C_{a, b}^{k}(x-y)=\int A_{a, b}^{k}(x-t) B^{k}(t-y) d^{2} t$, where $A^{k}$ and $B^{k}$ are defined by

$$
\begin{gathered}
A^{k}(p)=M^{-\frac{7}{2} k}\left(\zeta_{\varrho} \not p+m_{\varrho}\right) Q^{k}\left(p, m_{\varrho}, \zeta_{\varrho}\right)\left(p^{2}+16 M^{2 k+2}\right)^{-1} \\
\cdot\left[\left(p^{2}+M^{2 k+2}\right)\left(p^{2}+4 M^{2 k+2}\right)\left(p^{2}+8 M^{2 k+2}\right)\left(p^{2}+M^{2 k}\right)\left(p^{2}+4 M^{2 k}\right)\right]^{-1}, \\
B^{k}(p)=M^{\frac{7}{2} k}\left[\left(p^{2}+8 M^{2 k}\right)\left(p^{2}+16 M^{2 k}\right)\right]^{-1} .
\end{gathered}
$$

Similarly we write

$$
\bar{\psi}_{\varrho}=\bigoplus_{k=0, \ldots, \varrho} \bar{\psi}^{k} ; \quad \psi_{\varrho}=\bigoplus_{k=0, \ldots, \varrho} \psi^{k}
$$

the independent anticommuting variables $\bar{\psi}^{k}$ and $\psi^{k}$ being distributed according to the gaussian measure $d \mu^{k}$ of covariance $C^{k}$. Any external field $\bar{\psi}(y)$ or $\psi(z)$ is also decomposed as in (3.34); therefore any external variable $y$ or $z$ has an associated index $k(y)$ or $k(z)$; the external variables with index $k$ are written $y_{k}$ or $z_{k}$ for short. Also any vertex has 4 "fields" of given (possibly different) indices hooked to it. We define the index of the vertex as the highest of these 4 indices. More precisely we can write the interaction with cutoff $M^{k}$ in volume $\Gamma$ as:

$$
\left.V_{k}(\Gamma)=\int_{\Gamma}\left[\left(\underset{i=0, \ldots, k}{\bigoplus} \bar{\psi}^{i}(x)\right) \underset{i=0, \ldots, k}{\bigoplus} \psi^{i}(x)\right)\right]^{2} d^{2} x,
$$

and write $V^{k}(\Gamma)=V_{k}(\Gamma)-V_{k-1}(\Gamma)$. We need to develop $V^{k}$ as:

$$
V^{k}(\Gamma)=\sum_{k_{1}, k_{2}, k_{3}, k_{4}}^{k} \int_{\Gamma} \bar{\psi}_{k_{1}}(x) \psi_{k_{2}}(x) \bar{\psi}_{k_{3}}(x) \psi_{k_{4}}(x) d^{2} x,
$$

where the symbol $\sum^{k}$ means that $k_{i} \leqq k, \forall i \in\{1,2,3,4\}$ and $\exists i \in\{1,2,3,4\}$ such that $k_{i}=k$.

Let us introduce $\mathbf{D}_{k}$, the lattice of squares in $\Lambda$ of side $M^{-k}$, with centers of $M^{-k} \cdot \mathbb{Z}^{2}$; the lattice $\mathbf{D}_{k}$ is a refinement of $\mathbf{D}_{k-1}$. By the standard multinomial theorem, we may localize each vertex in a cube of the lattice corresponding to its index. If $\boldsymbol{\Delta}_{\varrho}(\Gamma)=\left\{(k, \Delta) \mid k \in[0, \varrho], \Delta \in \mathbf{D}_{k}, \Delta \in \Gamma\right\}$ to "localize" means simply to write:

$$
\sum_{n}\left[\lambda_{\varrho} V_{\varrho}(\Gamma)\right]^{n} / n !=\sum_{n(k, \Delta)} \prod_{(k, \Delta) \in \Delta}\left\{\left[\lambda_{\varrho} V^{k}(\Delta)\right]^{n(k, \Delta)} / n(k, \Delta) !\right\} .
$$

For each scale $k$, from $k=\varrho$ to $k=0$, we will perform first a cluster expansion relatively to the squares of $\mathbf{D}_{k}$, then a Mayer expansion of type (3.16)-(3.17); this is now a relatively standard technique [8-9]. Since the notation is heavy, we will just formalize the first two steps, then indicate how the final result can be described. At each scale these expansions are similar to the ones defined in detail in the preceding subsection, except that "connectedness" between two squares $\Delta$ and $\Delta^{\prime}$ of $\mathbf{D}_{k}$ can be created either by a derivation $d / d s_{b}, b \in \mathbf{B}^{k}=\left\{\right.$ pairs of squares of $\left.\mathbf{D}_{k}\right\}$, or by "Mayer 
graph" of higher index, containing $k$-frequency fields localized in these two squares. Therefore, let us introduce some new definitions: at scale $k$ the "true $k$ external variables" are the true external variables of indices $\geqq k$; the fields of indices $<k$ hooked to vertices of index $\geqq k$ will be called "low frequency variables" ("l.f.v."). More precisely, a low frequency variable of scale $k$ is a field $\bar{\psi}_{k^{\prime}}(x)$ or $\psi_{k^{\prime}}(\mathrm{x})$, with $k^{\prime} \leqq k-1$, together with the specification of an element of $\boldsymbol{\Delta}_{Q} \backslash \boldsymbol{\Delta}_{k-1}$ : the square of localization of the vertex corresponding to $x$.

At scale $\varrho$ the $\varrho$-polymers $\Gamma$ are made of the following list of objects: a set of squares of $\mathbf{D}_{\varrho}$, (whose union is called $\Gamma_{\varrho}$ ), plus possibly some of the true $\varrho$-external variables in them (called $y_{\varrho}(\Gamma), z_{\varrho}(\Gamma)$ ), plus a set of low-frequency variables of scale $\varrho$, called $\operatorname{LFV}(\Gamma)$. We call $N(\Gamma)$ the total number of external variables of $\Gamma$ (true external variables plus low frequency variables). The low frequency squares (1.f.s.) of $\Gamma$ are defined as the squares of $\mathbf{D}_{e-1}, \ldots, \mathbf{D}_{0}$ containing the l.f.v. of corresponding index (therefore, each such square has to contain at least one of the squares of $\Gamma$ in $\mathbf{D}_{\ell}$ ). The set of low frequency squares of $\Gamma$ in $\mathbf{D}_{k}$ is called $\operatorname{LFS}_{k}(\Gamma)$.

The amplitude of such a $\varrho$-polymer $\Gamma$ is computed by a formula similar to (3.8), but with $V_{\varrho}$ replaced by $V^{\varrho}$, and the constraints corresponding to the l.f.v. $\operatorname{LFV}(\Gamma)$ :

$$
\begin{aligned}
K_{\varrho}(\Gamma)= & \int \sum_{H \in \mathbf{B}_{e}^{e}(\Gamma)} \sum_{n(\varrho, \Delta)}\left(\prod_{b \notin H} I_{b}\right)\left(\prod_{b \in H} J_{b}\right) \prod \bar{\psi}^{\varrho}\left(y_{\varrho}(\Gamma)\right) \prod \psi^{\varrho}\left(z_{\varrho}(\Gamma)\right) \\
& \left.\cdot\left[\prod_{\Delta \subset \Gamma_{\varrho}}\left[\left(\lambda_{\varrho}\right) V^{\varrho}(\Delta)\right]^{n(\varrho, \Delta)} / n(\varrho, \Delta) !\right]\right|_{\operatorname{LFV}(\Gamma)} d \mu^{\varrho}(\{s\}),
\end{aligned}
$$

where $\mathbf{B}_{c}^{o}(\Gamma)$ is obviously the set of subsets of $\mathbf{B}^{o}\left\{=\left\{\right.\right.$ pairs of squares of $\left.\mathbf{D}_{\varrho}\right\}$ connecting together all squares of $\Gamma$, and the constraint $\left.\right|_{\mathrm{LFV}(\Gamma)}$ means that in the expansion (3.36) over all frequencies of $\prod_{\Delta \in \Gamma} V^{e}(\Delta)^{n(\varrho, \Delta)}$, the set of 1.f.v. obtained is precisely $\operatorname{LFV}(\Gamma)$. Then we can write:

$$
\begin{aligned}
\mathbf{S}_{2 p, \Lambda, \varrho}= & \sum_{k(y), k(z)} \sum_{n(k, \Delta), k \leqq \varrho-1} \int d \mu_{\varrho-1} \sum_{\text {disjoint }\left(\left\{y_{\ell}, z_{\varrho}\right\}\right)-\varrho \text {-Mayer graphs } \mathbf{M}_{\ell}} \mathbf{O} K_{\varrho}\left(\mathbf{M}_{\varrho}\right) \\
& \cdot \prod_{k(y) \leqq \varrho-1} \bar{\psi}^{k(y)}(y) \prod_{k(z) \leqq \varrho-1} \psi^{k(z)}(z) \prod_{(k, \Delta) \in \mathbf{\Delta}_{\varrho-1}}\left\{\left[\left(\lambda_{\varrho}\right) V^{k}(\Delta)\right]^{n(k, \Delta)} / n(k, \Delta) !\right\},
\end{aligned}
$$

where the $\varrho$-Mayer graphs $\mathbf{M}_{\varrho}$ are finite sequences of $\varrho$-polymers $\Gamma$, and $K_{\varrho}\left(\mathbf{M}_{\varrho}\right)$ is defined as in (3.12). LFS $\mathbf{S}_{k}\left(\mathbf{M}_{\varrho}\right)$ [respectively $\left.N\left(\mathbf{M}_{\ell}\right)\right]$ is defined as the union of the corresponding $\operatorname{LFS}_{k}(\Gamma)$ [respectively the sum of the corresponding $N(\Gamma)$ 's].

After the Mayer expansion, one obtains $\varrho$-super-Mayer graphs $\mathbf{S}_{\varrho}=\left(\mathbf{M}_{\varrho 1}, \ldots, \mathbf{M}_{\varrho}\right)$, "linear" in the $\varrho$-external variables with amplitudes $K_{\varrho}\left(\mathbf{S}_{\varrho}\right)$ $=\left[1 / r ! \prod_{k} K_{\varrho}^{T}\left(\mathbf{M}_{\varrho_{k}}\right)\right]\left[\right.$ with $K_{\varrho}^{T}(\mathbf{M}) \equiv T(\mathbf{M}) \cdot K_{\varrho}(\mathbf{M}), T(\mathbf{M})$ defined as in $\left.(3.15)\right]$, and corresponding definitions of $\operatorname{LFV}\left(\mathbf{S}_{\varrho}\right), \operatorname{LFS}_{k}\left(\mathbf{S}_{\varrho}\right)$, and $N\left(\mathbf{S}_{\varrho}\right)$. Then:

$$
\begin{aligned}
\mathbf{S}_{2 p, \Lambda, \varrho}= & \sum_{k(y), k(z)} \sum_{n(k, \Delta), k \leqq \varrho-1} \int d \mu_{\varrho-1} \sum_{\left.\left(y_{\varrho}, z_{\varrho}\right\}\right)-\varrho \text {-super-Mayer graphs } \mathbf{S}_{e}} \mathbf{O} K_{\varrho}\left(S_{\varrho}\right) \\
& \cdot \prod_{k(y) \leqq \varrho-1} \bar{\psi}^{k(y)}(y) \prod_{k(z) \leqq \varrho-1} \psi^{k(z)}(z) \prod_{(k, \Delta) \in \mathbf{\Delta}_{\varrho-1}}\left\{\left[\left(\lambda_{\varrho}\right) V^{k}(\Delta)\right]^{n(k, \Delta)} / n(k, \Delta) !\right\} .
\end{aligned}
$$

The ( $\varrho-1)$-polymers $\Gamma$ will be made of a set of squares of $\mathbf{D}_{\varrho^{-1}}$ (whose union is called $\left.\Gamma_{\varrho-1}\right)$, plus some of the $(\varrho-1)$-external variables in them, plus the $\varrho$-Mayer graphs $\mathbf{M}_{\varrho}$ in the super-Mayer graph $\mathbf{S}_{\varrho}$ such that $\mathrm{LFS}_{\varrho-1}\left(\mathbf{M}_{\varrho}\right)$ is contained in $\Gamma_{\varrho-1}$, 
plus a set of low frequency variables of scale $\varrho-1, \operatorname{LFV}(\Gamma)$. One should remark that in the ( $\varrho-1)$-cluster expansion, two squares $\Delta$ and $\Delta^{\prime}$ of $\mathbf{D}_{\varrho-1}$ can be linked either by an explicit derivation $d / d s_{b}, b=\left(\Delta, \Delta^{\prime}\right) \in \mathbf{B}_{\varrho-1}$, or by a $\varrho$-Mayer graph which has 1.f.v. of index $\varrho-1$ both in $\Delta$ and $\Delta^{\prime}$. Therefore, the formula for the amplitude of a $\varrho-1$ polymer $\Gamma$ is more complicated than (3.38):

$$
\begin{aligned}
K_{\varrho-1}(\Gamma)= & \int \sum_{H} \sum_{n(\varrho-1, \Delta)}\left(\prod_{b \notin H} I_{b}\right)\left(\prod_{b \in H} J_{b}\right) \prod \bar{\psi}^{\varrho-1}\left(y_{\varrho-1}(\Gamma)\right) \prod \psi^{\varrho-1}\left(z_{\varrho-1}(\Gamma)\right) \\
& \cdot \\
& \cdot\left(\left\{y_{\varrho}(\Gamma), z_{\varrho}(\Gamma)\right\}\right)-\varrho \text {-super-Mayer graphs } \mathbf{S}_{\varrho} \text { connecting the } H \text {-components of } \Gamma_{\varrho-1} K_{\varrho}\left(\mathbf{S}_{\varrho}\right) \\
& \left.\cdot\left[\prod_{\Delta \in \Gamma_{\varrho-1}}\left[\left(\lambda_{\varrho}\right) V^{\varrho-1}(\Delta)\right]^{n(\varrho-1, \Delta)} / n(\varrho-1, \Delta) !\right]\right|_{\operatorname{LFV}(\Gamma), N(\Gamma)} d \mu^{\varrho-1}(\{s\}),(3.41)
\end{aligned}
$$

where the sums are performed over set of bonds $H$ which are pairs of squares of $\Gamma_{\varrho-1}$, but which do not any more connect necessarily $\Gamma_{\varrho-1}$, and over super-Mayer graphs $\mathbf{S}_{\varrho}$ which have their $(\varrho-1)$-1.f.v. in $\Gamma_{\varrho-1}$ and which "achieve" the connection of $\Gamma_{\varrho-1}$. This means that given $H$, the connected components of $\Gamma_{\varrho-1}$ linked by bonds of $H$ [called $H$-components in (3.41)] are themselves connected together by Mayer graphs of $\mathbf{S}_{\varrho}$.

After the $\varrho-1$ cluster expansion we perform the $(\varrho-1)$-Mayer expansion and obtain $(\varrho-1)$-Mayer graphs and $(\varrho-1)$-super-Mayer graphs, etc.... At the end of the 0-Mayer expansion, we obtain 0-super-Mayer graphs which are "linear" in the true external variables. The normalization $Z_{\Lambda, \varrho}$ can be factorized at this stage and $S_{2 p, \Lambda, \varrho}$ or $S_{2 p, \Lambda, \varrho}^{T}$ computed as in (3.18)-(3.19). Then the thermodynamic limit can be performed just as in Lemma 3.1. From now on, for simplicity, we will work only in this thermodynamic limit, hence on the normalized functions $S_{2 p, \varrho}$ and $S_{2 p, \varrho}^{T}$ defined in (3.21)-(3.22). Let us recall that these functions are defined, for $\lambda_{\varrho}$ small enough, by the phase space expansion, which by Lemma 3.1 is an absolutely convergent expansion in powers of $\lambda_{\varrho}$. Indeed by identification order by order, the sum for instance of the 0-Mayer graphs linear in all external variables with total number of vertices $n$ in the phase space expansion must be equal to the sum of the ordinary Mayer graphs with $n$ vertices in the single Mayer expansion, and also to the sum of all connected Feynman graphs containing $n$ vertices and all the external variables; since these three expressions are the $n^{\text {th }}$ order of perturbation theory for the truncated Schwinger functions $S_{2 p, \varrho}^{T}$. The reader may therefore think of the Mayer graphs as just a reorganization of Feynman graphs in a way which exhibits the cancellations between them responsible for convergence of the bare perturbation series. It is also useful to remark that the sum of the $k$-Mayer graphs at a given order with a prescribed set of external variables ("true" or "low-frequency") is the same quantity as the sum of the connected Feynman graphs of same order and same set of external variables, with propagators $\sum_{j=k, \ldots, \varrho} C^{j}$ instead of $C_{\varrho}$. In particular it is translation invariant, and does not depend on the particular choice of the lattice of cubes $\mathbf{D}$. In the next section we will perform one last reorganization of the perturbation expansion: the phase space expansion will be reshuffled into a "partly renormalized phase space expansion" (PRPSE), expressed in terms of "effective constants." It is the crucial step, since in contrast with the previous ones, it involves combining together different orders of the ordinary perturbation theory, to define the effective constants. (In fact, in the way we define it, it involves 
infinite (but absolutely convergent) resummations of some pieces of perturbation theory.) This PRPSE is the tool which allows us to improve sloppy estimates like those of Lemma 3.1, and to achieve our construction.

Let us include some comments on the interest of the PRPSE; it lies "between" the bare and the renormalized expansion since the divergent graphs are renormalized (i.e., combined with their counterterms) if and only if their internal momenta are higher than their external momenta. It is easy to verify that this is precisely the case, where the renormalization is "useful," i.e., where it has a positive net effect (see, e.g., [16]). Therefore, the PRPSE is optimal from the point of view of getting sharp estimates; the only price to pay is that it is a power series in an infinite number of "effective constants" instead of a single one. Since it lies "in between," the PRPSE can be obtained equally well from the bare or from the renormalized expansion; in the first case (which is the strategy used in this paper and for example in [9]) one adds the "useful" part of the counterterms to the bare expansion to partly renormalize it and one subtracts this same "useful" part from the bare constants to get effective constants; in the second case (strategy followed for example in [6]) one simply absorbs the "useless" part of the counterterms in the coupling constants by adding it to the renormalized constants, again obtaining the same expansion in terms of effective constants.

\section{The Renormalization}

\section{The "Partly Renormalized" Expansion}

We will now transform the phase space expansion of a given Schwinger function into a "partly renormalized phase space expansion" (in short PRPSE). This is done inductively, replacing after the $k^{\text {th }}$ Mayer expansion in the preceding subsection the amplitudes of all the " $k$-quadrupeds" and " $k$-bipeds" by the sum of a "renormalized amplitude" and of a "counterterm." At each scale $k$ we define the " $k$ quadrupeds" $Q_{k}$ (respectively the " $k$-bipeds" $B_{k}$ ) as the $k$-Mayer graphs with $N=4$ external legs all low frequency variables (respectively with $N=2$ ), which are of the type $(\bar{\psi} \cdot \psi)^{2}$ [respectively $\left.(\bar{\psi} \cdot \psi)\right]$ (see Lemma 2.1), and which have at least one internal leg of frequency $k$. For simplicity we may forget from now on to distinguish between $\bar{\psi}$ and $\psi$ when it is unimportant. Let us again sketch the first step, then give the general induction rule.

The amplitude $K_{\varrho}^{T}\left(Q_{\varrho}\right)$ of a $\varrho$-quadruped $Q_{\varrho}$ is defined using (3.38) and $K_{\varrho}^{T}\left(Q_{\varrho}\right)$ $=\sum_{\mathbf{M}} T(\mathbf{M}) K_{\varrho}(\mathbf{M})$; the 4 external fields will be called $\psi_{k_{1}}\left(x_{1}\right), \psi_{k_{2}}\left(x_{2}\right), \psi_{k_{3}}\left(x_{3}\right)$, $\psi_{k_{4}}\left(x_{4}\right)$, and we recall that $x_{1}, x_{2}, x_{3}$, and $x_{4}$ are integrated over their localization squares, called $\Delta_{1}, \Delta_{2}, \Delta_{3}$, and $\Delta_{4}$, which in this case are in $\mathbf{D}_{\varrho^{\circ}}$. Note that some of the $x_{i}$ 's may coincide, since different external fields may be hooked to the same vertex, and some of the $\Delta_{i}$ 's may also coincide. We define:

$$
\begin{gathered}
\delta Q_{\varrho}^{i}=\left[1 /\left|\Delta_{i}\right|\right] K_{\varrho}^{T, A}\left(Q_{\varrho}\right), \\
\delta \lambda_{\varrho}=\sum_{Q_{\varrho} / 0 \in \Delta_{i}} \delta Q_{\varrho}^{i},
\end{gathered}
$$


where in (4.1) the "amputated activity" $K_{\varrho}^{T, A}\left(Q_{\varrho}\right)$ is defined by suppressing in the definition of $K_{\varrho}^{T}\left(Q_{\varrho}\right)$ the product of the external fields $\psi_{k_{1}}\left(x_{1}\right) \psi_{k_{2}}\left(x_{2}\right) \psi_{k_{3}}\left(x_{3}\right) \psi_{k_{4}}\left(x_{4}\right)$. We recall, again, that in (3.38), hence in (4.1), $x_{i}$ is integrated over its localization square $\Delta_{i}$. By translation invariance, $\delta \lambda_{\varrho}$ is independent of the choice of $i$ and of the origin 0 in (4.2). We remark that in Fourier space, $\delta \lambda_{\varrho}$ is the sum of the values of the $\varrho$-quadrupeds at 0 external momenta, this is the most usual prescription in order to renormalize theories with a non-zero bare mass. By (3.19) we see that in the usual language of perturbation theory, $\delta \lambda_{\varrho}$ is exactly the sum of the connected 4-point Feynman graphs at 0 external momenta and internal propagators in the slice $\varrho$.

The renormalized amplitude $K_{\varrho}^{T, R}\left(Q_{\varrho}\right)$ is defined by:

$$
\begin{aligned}
K_{\varrho}^{T, R}\left(Q_{\varrho}\right) \equiv & K_{\varrho}^{T}\left(Q_{\varrho}\right)-(1 / 4)\left\{\sum_{i=1, \ldots, 4} \delta Q_{\varrho}^{i} \psi_{k_{1}}\left(x_{i}\right) \psi_{k_{2}}\left(x_{i}\right) \psi_{k_{3}}\left(x_{i}\right) \psi_{k_{4}}\left(x_{i}\right)\right\} \\
= & (1 / 4)\left\{K_{\varrho}^{T, A}\left(Q_{\varrho}\right) \sum_{i=1, \ldots, 4} \sum_{j \neq i}\left[\prod_{j^{\prime}<j} \psi_{k_{J}}\left(x_{i}\right)\right]\right. \\
& \left.\cdot\left(\psi_{k_{j}}\left(x_{j}\right)-\psi_{k_{j}}\left(x_{i}\right)\right)\left[\prod_{j^{\prime}>j} \psi_{k_{j}}\left(x_{j^{\prime}}\right)\right]\right\},
\end{aligned}
$$

where again our convention is that the integration signs for the $x_{i}$ 's are hidden in the formulas for $K^{T}, K^{T, A}$, and $\delta Q_{\varrho}^{i}$. The differences $\psi_{k_{j}}\left(x_{j}\right)-\psi_{k_{j}}\left(x_{i}\right)$ are systematically written as "gradients" acting on the external fields:

$$
\psi_{k_{j}}\left(x_{j}\right)-\psi_{k_{j}}\left(x_{i}\right)=\int_{0}^{1} d \theta\left(x_{j}-x_{i}\right) \cdot \nabla \psi_{k_{j}}\left[x_{i}+\theta\left(x_{j}-x_{i}\right)\right],
$$

and after integration with respect to $d \mu^{k_{j}}$, the gradient will act on the corresponding "legs" $A$ or $B$.

Similarly we define, for any $\varrho$-biped $B_{\varrho}$, counterterms $\delta B_{\varrho}$ for the corresponding contributions to the mass and wave function renormalizations, and a renormalized amplitude $K_{\varrho}^{T, R}\left(B_{\varrho}\right)$, which has $t w o$ gradients acting on its external fields (this requires some easy algebra similar to that of Lemma 2.1), and we define:

$$
\lambda_{\varrho-1}=\lambda_{\varrho}+\delta \lambda_{\varrho} ; \quad m_{\varrho-1}=m_{\varrho}+\delta m_{\varrho} ; \quad \zeta_{\varrho-1}=\zeta_{\varrho}+\delta \zeta_{\varrho},
$$

where $\delta m_{\varrho}$ and $\delta \zeta_{\varrho}$ are defined by formulas similar to (4.2).

With some easy combinatoric checks, one can rewrite now the formula (3.40) expressing the phase space expansion after the $\varrho^{\text {th }}$ step with the following rules:

- all the $\varrho$-quadrupeds and $\varrho$-bipeds are renormalized, i.e., their amplitude is replaced by the renormalized amplitude (4.4);

- the interaction $\left[\left(\lambda_{\varrho}\right) V^{k}(\Delta)\right]^{n(k, \Delta)} / n(k, \Delta)$ ! [see (3.37)], $k<\varrho$, is replaced by $\left[\left(\lambda_{\varrho-1}\right) V^{k}(\Delta)\right]^{n(k, \Delta)} / n(k, \Delta) !$.

- the gaussian measure $d \mu_{\varrho-1}$ corresponds now to an effective propagator:

$$
C_{\varrho-1}^{*}(p) \equiv \eta_{\varrho-1}(p) \cdot\left[\zeta_{\varrho-1}(p) \not p+m_{\varrho-1}(p)\right] /\left[\zeta_{\varrho-1}^{2}(p) p^{2}+m_{\varrho-1}^{2}(p)\right],
$$

where

$$
\zeta_{\varrho-1}(p) \equiv \zeta_{\varrho}\left(1-\eta_{\varrho-1}(p)\right)+\zeta_{\varrho-1}\left(\eta_{\varrho-1}(p)\right)
$$

and

$$
m_{\varrho-1}(p) \equiv m_{\varrho}\left(1-\eta_{\varrho-1}(p)\right)+m_{\varrho-1}\left(\eta_{\varrho-1}(p)\right)
$$


[Hint: to find formula (4.6) for the effective propagator, we remark that after renormalization of the $\varrho$-bipeds, one is left with an expansion with propagators $C_{\varrho-1}$ which are dressed with arbitrary numbers of mass and wave function insertions. The dressed propagator $C_{\varrho-1}^{*}$ is therefore the sum of the geometric series:

$$
\sum_{i \geqq 0}\left[\left(\delta \zeta_{\varrho} \not-\delta m_{\varrho}\right)\right]^{i}\left[\eta_{\varrho-1}(p)\left(\zeta_{\varrho} \not p+m_{\varrho}\right) /\left(\zeta_{\varrho}^{2} p^{2}+m_{\varrho}^{2}\right)\right]^{i+1},
$$

which is nothing but (4.6).]

We can now rewrite $C_{\varrho-1}^{*}=C^{* \varrho-1}+C_{\varrho-2}^{*}$, with:

$$
\begin{aligned}
C^{* \varrho-1}(p) \equiv & {\left[\eta_{\varrho-1}(p)-\eta_{\varrho-2}(p)\right] } \\
& \cdot\left[\zeta_{\varrho-1}(p) \not p+m_{\varrho-1}(p)\right] /\left[\zeta_{\varrho-1}^{2}(p) p^{2}+m_{\varrho-1}^{2}(p)\right]
\end{aligned}
$$

and generalize inductively this analysis to any slice $k$. We obtain the full PRPSE, which is identical to the phase space expansion of the preceding section with the following rules:

- all the quadrupeds and bipeds are renormalized, i.e., their amplitude is replaced by the renormalized amplitude;

- the interaction $\left[\left(\lambda_{\varrho}\right) V^{k}(\Delta)\right]^{n(k, \Delta)} / n(k, \Delta)$ ! [see (3.37)], $k>\varrho$, is replaced by $\left[\left(\lambda_{k}\right) V^{k}(\Delta)\right]^{n(k, \Delta)} / n(k, \Delta) !$

- the propagator in the slice $k$ is no longer $C^{k}$ but $C^{* k}(p)$, defined as:

$$
C^{* k}(p) \equiv \eta_{k}(p) \cdot\left[\zeta_{k}(p) p+m_{k}(p)\right] /\left[\zeta_{k}^{2}(p) p^{2}+m_{k}^{2}(p)\right],
$$

where

$$
\zeta_{k}(p) \equiv \zeta_{\varrho}\left(1-\eta_{\varrho-1}(p)\right)+\sum_{k<i<\varrho}\left[\zeta_{i}\left(\eta_{i}(p)-\eta_{i-1}(p)\right)\right]+\zeta_{k} \eta_{k}(p)
$$

and

$$
m_{k}(p) \equiv m_{\varrho}\left(1-\eta_{\varrho-1}(p)\right)+\sum_{k<i<\varrho}\left[m_{i}\left(\eta_{i}(p)-\eta_{i-1}(p)\right)\right]+m_{k} \eta_{k}(p) .
$$

As expected, $\lambda_{k}, m_{k}$, and $\zeta_{k}$ are defined inductively by the formula:

$$
\lambda_{k}=\lambda_{k+1}+\delta \lambda_{k+1} ; \quad m_{k}=m_{k+1}+\delta m_{k+1} ; \quad \zeta_{k}=\zeta_{k+1}+\delta \zeta_{k+1}
$$

where $\delta \lambda_{k+1}, \delta m_{k+1}$, and $\delta \zeta_{k+1}$ are of course given by the sum of the counterterms corresponding to $(k+1)$-quadrupeds and bipeds, computed with the effective couplings $\lambda_{k^{\prime}}$ and the effective propagators $C^{* k^{\prime}}, k^{\prime} \geqq k+1$.

\section{The Behavior of the Effective Constants}

We will study precisely the evolution of our effective constants as functions of $k$. We concentrate our efforts on the coupling constant behavior [under the ansatz (2.21)-(2.22)]. (Other approaches are possible, see Subsect.IV.3). The main behavior of $\lambda_{k}$ is governed by asymptotic freedom, but the "subleading" term also plays some rôle. The next four lemmas will be proven inductively under the ansatz (1.1) on $\lambda_{\varrho}$; more precisely Lemmas $4.3,4.4,4.1$, and 4.2 are proved in this order, successively for $k=\varrho, \varrho-1, \ldots$, up to $k=0$. 
We may decompose the sum (4.2) as:

$\delta \lambda_{k}=\sum_{k \text {-quadrupeds } Q_{k} \text { with at most } 3 \text { vertices }} \delta Q_{k}+\sum_{k \text {-quadrupeds } Q_{k} \text { with at least } 4 \text { vertices }} \delta Q_{k}$.

\section{Lemma 4.1}

$$
\delta \lambda_{k}=-(\log M)\left[\beta_{2}^{k, \varrho} \lambda_{k}^{2}+\left(\gamma_{3}^{k, \varrho}-\left(\beta_{2}^{k, \varrho}\right)^{2}(\log M)\right) \lambda_{k}^{3}+O\left(\lambda_{k}^{4}\right)\right],
$$

where $\beta_{2}^{k, \varrho}$ and $\gamma_{3}^{k, \varrho}$ are defined (using obvious graphical notation) by:

$$
\begin{gathered}
(\log M) \beta^{k, \varrho}=\sum_{i_{1}, i_{2}=k, \ldots, \varrho \text { with inf }\left\{i_{1}, i_{2}\right\}=k} \int_{\sum_{i_{2}}} \sum_{y} d^{2} y \\
(\log M) \gamma_{3}^{k, \varrho}=\sum_{i_{1}, i_{2}, i_{3}, i_{4}=k, \ldots, \varrho \text { with } \inf \left\{i_{1}, i_{2}, i_{3}, i_{4}\right\}=k}^{i_{1}}
\end{gathered}
$$

and the sign $R$ means that the inner bubble is renormalized; Furthermore the following limits exists:

$$
\beta_{2}=\lim _{k \rightarrow \infty} \beta_{2}^{k, \infty}, \quad \gamma_{3}=\lim _{k \rightarrow \infty} \gamma_{3}^{k, \infty}
$$

and $\beta_{2}$ is the usual first coefficient of the $\beta$ function, computed with wave function $\zeta=1$, introduced in (2.17). We remark that with the standard convention $\beta_{2}$ is negative, which corresponds to asymptotic freedom, hence to $\delta \lambda_{k}>0$ for small $\lambda_{k}$. Proof. We have, with graphical notation:

$$
\begin{aligned}
& \delta \lambda_{k}=\sum_{i_{1}, i_{2}=k, \ldots, \varrho \text { with } \inf \left\{i_{1}, i_{2}\right\}=k} \lambda_{i_{1,2}}^{2} \int \overbrace{i_{2}}^{i_{1}} d^{2} y \\
& +\sum_{i_{1}, i_{2}, i_{3}, i_{4}=k, \ldots, \varrho \text { with } \inf \left\{i_{1}, i_{2}, i_{3}, i_{4}\right\}=k} \lambda_{i_{1,2}} \lambda_{i_{1,3}, 4} \lambda_{i_{2,3}, 4} \int C_{z}^{i_{2}} R_{c}^{y} d_{i_{4}} d^{2} y d^{2} z \\
& +\lambda_{i_{1,2}, 2} \lambda_{i_{1,2}, 4,4} \lambda_{i_{3,4}} \int x_{i_{2}}^{i_{1}} \underset{y}{\longrightarrow} \underbrace{i_{3}}_{i_{4}} d^{2} y d^{2} z+7 \rho+\gamma \\
& +\succ \odot \sum_{k \text {-quadrupeds } Q_{k} \text { with at least 4vertices }} \delta Q_{k} \text {, }
\end{aligned}
$$

where $i_{1,2}=\sup \left\{i_{1}, i_{2}\right\}$ etc...; and the symbol $R_{c}$ means that the inner bubble is renormalized if the condition $\inf \left\{i_{3}, i_{4}\right\}>\sup \left\{i_{1}, i_{2}\right\}$ holds; the contributions to $\delta \lambda_{k}$ of the graphs $>0,>00,>\circlearrowright$ are of order $M^{-k}$ (either we can use parity considerations or using translation invariance one at least of the external field get a gradient and the graph doesn't contribute to $\delta \lambda_{k}$.) 
We transform $\lambda_{i}, \lambda_{i_{1,2}}, \lambda_{i_{1,3}, 4}$, and $\lambda_{i_{2,3,4}}$ into $\lambda_{k}$ using the recursive relation (4.8), and keeping all terms of order at most 3 in $\lambda_{k}$. In this process, one can verify that the counterterms necessary to replace the conditional sign $R_{c}$ by a sign $R$ in (4.14) are exactly generated once, except for one subtlety: the counterterm when $i_{1}$ or $i_{2}=i_{3}$ or $i_{4}=k$ is missing. But it is precisely equal to $\left(\beta_{2}^{k, \varrho}\right)^{2}$ and this explains the presence of the corresponding term in (4.10). The terms of order 4 or more in $\lambda_{k}$ generated in the process and the contributions of Mayer graphs of order 4 or more may be estimated uniformly by $O\left(\lambda_{k}^{4}\right)$ as a consequence of the bounds of the next subsection, and of the fact that

$$
\lambda_{k}=\left(1 /-\beta_{2}(\log M) k\right)(1+O[(\log k) / k])
$$

(see Lemma 4.3 at order $k$ ) (but there is no logical bug, since as remarked above, the proof of Lemmas 4.1-4.4 is inductive).

One has that:

$$
\lim _{k, \varrho \rightarrow \infty} \sum_{i_{1}, i_{2} \geqq k, \text { with inf }\left\{i_{1}, i_{2}\right\}=k} f\left(i_{1}, i_{2}\right)=-\beta_{2} \ln M,
$$

with

$$
\begin{aligned}
& f\left(i_{1}, i_{2}\right)=\int \chi_{i_{2}}^{i_{1}} \chi_{y} d^{2} y, \\
& \lim _{k, \varrho \rightarrow \infty} \sum_{i_{1}, i_{2}, i_{3}, i_{4} \geqq k, \text { withinf }\left\{i_{1}, i_{2}, i_{3}, i_{4}\right\}=k} f\left(i_{1}, i_{2}, i_{3}, i_{4}\right)=-\gamma_{3} \ln M,
\end{aligned}
$$

with

$$
f\left(i_{i}, i_{2}, i_{3}, i_{4}\right)=\int C_{i_{2}}^{i_{3}}{ }_{i_{z}}^{i_{1}} d^{2} y d^{2} z .
$$

To prove it one uses the fact that for all $p: f\left(i_{1}, \ldots, i_{r}\right)=f\left(i_{1}-p, \ldots, i_{r}-p\right)$ up to corrections which go to zero as $\varrho$ and the $i$ 's go to infinity. Then in the above sums one can fix by translation $i_{1}$ to be equal to $k$ and there are now no more conditions on the other $i$ 's; thus $-\beta_{2}$ and $-\gamma_{3}$ are equal to: $(q)$ [the value of the corresponding diagram (with no cutoff and no mass), where the modulus of one momentum is kept fixed equal to $q$ ].

These are the values of the Callan-Symanzik coefficients and have been computed for this model, see $[1,2,23]$.

It remains to verify (4.13). Here a subtlety arises. In the definition (4.11) of $\beta_{2}^{k, \varrho}$ the lines with indices $i$ and $k$ have effective propagators $C^{* i}$ and $C^{* k}$. To relate $\beta_{2}^{k, \varrho}$ to $\beta_{2}$ we need to reexpress the wave function $\zeta_{i}$ or $\zeta_{k}$ in terms of $\zeta_{\varrho}=1$. Substituting the value (4.22) for $\zeta_{k}$ obtained in Lemma 4.4 into (4.11), we obtain that:

$$
\beta_{2}^{k, \varrho}=\beta_{2}+\delta^{k, \varrho}+\lambda_{k} \delta_{3}^{k, \varrho}-\left[\delta_{3}^{k, \varrho} / \varrho\left(-\beta_{2} \log M\right)\right]+O\left((\log k) / k^{2}\right),
$$

where again $\delta_{3}=\lim _{k \rightarrow \infty} \delta_{3}^{k, \infty}$ exists, $\delta_{3}$ is a constant computable in terms of $\gamma_{2}$ and $\beta_{2}$ whose precise value is irrelevant, and:

$$
\begin{gathered}
\delta^{k, \varrho} \leqq e^{-O(1) k}+e^{-O(1)(\varrho-k)} \\
\left|\delta^{k, \varrho}-\delta^{k, \varrho+1}\right| \leqq e^{-O(1)(\varrho-k)} ; \quad\left|\delta^{k, \varrho}-\delta^{k+1, \varrho+1}\right| \leqq e^{-O(1) k} .
\end{gathered}
$$


In (4.15) the term $O\left(\log k / k^{2}\right)$ is an upper bound on all the terms of order two or more in $(1 / k)$ or $(1 / \varrho)$ with possible logarithms of $k$ and $\varrho$ arising from the development of $\lambda_{k}=\left(1 /-\beta_{2} k\right)(1+O[(\log k) / k])$. The first bound in (4.16) corresponds to the difference between the exponentially converging sum over $i$ in (4.11) and its limit, which gives the $e^{-O(1)(\varrho-k)}$ term, and to the existence of a mass which at low energy gives a correction to the scale invariance of $\beta_{2}^{k, \varrho}$; this correction is bounded by $e^{-O(1) k}$. Finally, in the differences $\left|\delta^{k, \varrho}-\delta^{k, \varrho+1}\right|$ and $\left|\delta^{k, \varrho}-\delta^{k+1, \varrho+1}\right|$ there is, respectively, no mass and no u.v. cutoff correction, which achieves the proof of (4.16). For this analysis, we use the "exponential decay in momentum space" (in the separation of slices) of the PRPSE (see [16] and the next section); the estimate on the mass correction requires also that under the ansatz (2.24) the running mass $m_{k}$ remains bounded for any $k$ up to $k=0$ by $O(1)$, which is true by Lemma 4.4 (again no logical loop!).

Combining Lemma 4.1 with (4.15) and a similar tedious but straightforward analysis for the $\gamma_{3}^{k, o}$ term in (4.10), and remarking that the $e^{-O(1) k}$ term in (4.16) can be absorbed in the $O\left(\log k / k^{2}\right)$ term in (4.15). One obtains the following more precise statement (always under the ansatz (2.24) and (1.1):

\section{Lemma 4.2.}

$$
\begin{aligned}
\delta \lambda_{k}=-(\log M) & {\left[\beta_{2} \lambda_{k}^{2}+\left(\beta_{3}-\beta_{2}^{2}(\log M)\right) \lambda_{k}^{3}-\delta_{3} \lambda_{k}^{2} / \varrho\left(-\beta_{2} \log M\right)\right.} \\
+ & \left.O\left(\log k / k^{4}\right)+\left(\lambda_{k}^{2}\right)\left(e^{-O(1)(\varrho-k)}\right)\right]
\end{aligned}
$$

where by definition $\beta_{3}=\gamma_{3}+\delta_{3}$ is the usual third order term in the $\beta$-function used in (2.17) and (2.20).

The next lemma controls the behavior of $\lambda_{k}$ under an appropriate condition on $\lambda_{\varrho}$ :

Lemma 4.3. There exists some constant $O(1)$ such that if $C$ is a positive number satisfying $C \geqq O(1)$, and $\lambda_{\varrho}$ is defined by (1.1), then $\lambda_{k}$ is positive and behaves according to the bounds:

$$
\begin{array}{r}
-\beta_{2}(\log M) k+\left(\beta_{3} / \beta_{2}\right) \log k+C / 2 \leqq \lambda_{k}^{-1} \\
\leqq-\beta_{2}(\log M) k+\left(\beta_{3} / \beta_{2}\right) \log k+2 C .
\end{array}
$$

Proof. Assuming (4.18) at order $k$ rewrite (4.8) and (4.17) as:

$$
\begin{aligned}
\lambda_{k-1}^{-1}-\lambda_{k}^{-1}= & (\log M)\left[\beta_{2}+\beta_{3} /\left(-\beta_{2}(\log M) k\right)\right. \\
& \left.-\delta_{3} / \varrho\left(-\beta_{2} \log M\right)+O\left((\log k) / k^{2}\right)+e^{-O(1)(\varrho-k)}\right],
\end{aligned}
$$

and (4.18) will follow from the logarithmic divergence of $1 / k$, the summability of $(\log k) / k^{2}, e^{-O(1)(\varrho-k)}$, and the obvious bound

$$
\sum_{j=k, \ldots, \varrho}\left(\left|\delta_{3}\right| / \varrho\right)=\left|\delta_{3}\right|(\varrho-k) / \varrho \leqq\left|\delta_{3}\right| .
$$

Lemma 4.4. Under the ansatz (2.21)-(2.22) and (1.1) the running mass and wave function satisfy:

$$
\begin{gathered}
(m / 2) \cdot k^{-(N-1 / 2) /(N-1)} \leqq m_{k} \leqq 2 m \cdot k^{-(N-1 / 2) /(N-1)} \\
\zeta_{k}-\zeta_{\varrho}=\left(\gamma_{2} / \beta_{2}^{2} \log M\right)[(1 / k)-(1 / \varrho)]+O\left[(\log k) / k^{2}\right] .
\end{gathered}
$$


Proof. We write a first order perturbation analysis for $\delta m_{k}$ and a second order analysis for $\delta \zeta_{k}$ similar to the third order analysis for $\delta \lambda_{k}$ in (4.9)-(4.10), and the results follows easily. For instance $\delta \zeta_{k}$ may be estimated by:

$$
\delta \zeta_{k}=\left(\gamma_{2} / \beta_{2}^{2} \log M\right) / k^{2}+O\left[(\log k) / k^{3}\right]+\left[e^{-O(1)(\varrho-k)}\right] / k^{2}
$$

from which it is elementary to derive (4.21).

We are ready to state our main results:

Theorem IV.1. Under the ansatz (2.21)-(2.22) and (1.1), the PRPSE is absolutely and uniformly convergent for $C$ positive large enough. If $S_{2 p, \varrho}(C)$ are the normalized Schwinger function sums of this PRPSE, then the limits $S_{2 p}(C)=\lim _{\varrho \rightarrow \infty} S_{2 p, \varrho}(C)$ exist and are the Schwinger functions of a non-trivial Euclidean theory satisfying The Osterwalder-Schrader axioms.

Theorem IV.2. Under the conditions of Theorem IV.1, the limits $S_{2 p}(C)$ $=\lim _{\varrho \rightarrow \infty} S_{2 p, \varrho}(C)$ also exist and are analytic in $C$ for $C$ complex with $\operatorname{Re} C \geqq O(1)$. The renormalized coupling constant $\lambda_{\text {ren }}(C)$ for the $\mathrm{BPHZ}$ scheme at 0 momenta corresponding to this theory lies in a disk $D_{C^{\prime}}=\left\{\gamma / \operatorname{Re}(1 / \lambda)>C^{\prime}\right\}$. For $C^{\prime}$ large enough and $\lambda_{\text {ren }} \in D_{C^{\prime}}$, the map from $C$ to $\lambda_{\text {ren }}$ may be inverted, and $C$ becomes an analytic function of $\lambda_{\text {ren }} \in D_{C^{\prime}}$, with $\operatorname{Re} C \geqq O(1)$. Therefore, the theory can be parametrized now by $\lambda_{\text {ren }}$ instead of $C$, is analytic in $D_{C^{\prime}}$, and is the Borel sum of the usual BPHZ renormalized series in $\lambda_{\text {ren }}$ with renormalized mass and wave function $m_{\text {ren }}$ and $\zeta_{\text {ren }}$ which are finite non-zero functions of $m$ and $\lambda_{\mathrm{ren}}$.

Remark. It should be understood that in the BPHZ prescription, the 4-point terms which are not of the type $(\bar{\psi} \psi)^{2}$, are not renormalized (they are convergent, see Lemma 11.1).

The proof of these two Theorems follows from the results of the two next sections. We will prove in Sect. V that under the condition (1.1) the PRPSE is absolutely convergent. Our estimates will be uniform in $\varrho$. Pushing the analysis one order further will enable us to check that the running parameters $\lambda_{k}^{\varrho}, m_{k}^{o}$, and $\zeta_{k}^{\varrho}$ computed with the ansatz (2.21)-(2.22) and (1.1) converge to finite values $\lambda_{k}^{\infty}, m_{k}^{\infty}$, and $\zeta_{k}^{\infty}$ as $\varrho \rightarrow \infty$. This will prove the second part of Theorem IV.1, and give a computable expansion, (according to the criteria of [17]) for the Schwinger functions $S_{2 p}(C)$; they are the sums of the PRPSE, with no cutoff, effective vertices $\lambda_{k}^{\infty}$ and effective propagators $C^{* k, \infty}(p)$ defined by:

$$
C^{* k, \infty}(p) \equiv \eta_{k}(p) \cdot\left[\zeta_{k}^{\infty}(p) \not p+m_{k}^{\infty}(p)\right] /\left\{\left[\zeta_{k}^{\infty}(p)\right]^{2} p^{2}+\left(m_{k}^{\infty}\right)^{2}(p)\right]
$$

with:

$$
\zeta_{k}^{\infty}(p) \equiv \sum_{k<i}\left[\zeta_{i}^{\infty}\left(\eta_{i}(p)-\eta_{i-1}(p)\right)\right]+\zeta_{k}^{\infty} \eta_{k}(p)
$$

and:

$$
m_{k}^{\infty}(p) \equiv \sum_{k<i}\left[m_{i}^{\infty}\left(\eta_{i}(p)-\eta_{i-1}(p)\right)\right]+m_{k}^{\infty} \eta_{k}(p)
$$


In Sect. VI the relationship of the theory to its usual perturbative renormalized series is analyzed and Theorem IV.2 is proved. But Borel summability is the key to a particularly simple verification of the axioms. indeed the reader may easily convince himself that the construction of this paper could have been repeated with an other form of the upper cutoff $\varrho$, for instance a lattice cutoff. The limits $S_{2 p}^{\text {P.V. }}(C)$ (respectively, $S_{2 p}^{\text {lattice }}(C)$ ) obtained with the Pauli-Villars cutoff (respectively the "lattice" cutoff) satisfy all the O.S. axioms except O.S. positivity (respectively Euclidean invariance); indeed this is the only axiom violated by the Pauli-Villars cutoff (respectively the lattice cutoff). But these two limits are equal, since they are the Borel sum of the same series! Therefore, they satisfy all the O.S. axioms, which achieves the proof of Theorem IV.1.

\section{Additional Remarks}

There are other equivalent presentations of the results of the preceding subsection which ultimately yield to the same continuum theory. Here are some attractive possibilities:

- The ansatz (2.22) could be replaced by the ansatz used in [13]:

$$
\zeta_{\varrho}=1+\gamma_{2} /\left(\beta_{2}^{2} \varrho \log M\right) \text {. }
$$

The advantage is that the term $\gamma_{3}^{\prime} \lambda_{k}^{2} / \varrho$ in (4.17) disappears. The price to pay is the use of an ansatz for $\zeta_{\varrho}$ which is not constant in $\varrho$, although in fact there is no infinite wave function renormalization in the theory.

- One might work in the reverse order, keeping $\lambda_{\text {ren }}, m_{\text {ren }}$, and $\zeta_{\text {ren }}$ defined by the BPHZ conditions fixed, and adjusting $\lambda_{\varrho}, m_{\varrho}$, and $\zeta_{\varrho}$ at each step. The advantage is that the theory is directly parametrized by renormalized constants which, instead of our $m$ and $C$, correspond to a well defined scheme. The price to pay is that $\lambda_{\varrho}, m_{\varrho}$, and $\zeta_{\varrho}$ are given by complicated formulas. A particularly attractive possibility (see also the introduction) is to work from the beginning with the PRPSE' of Sect. VI, hence with $\lambda_{\varrho}, m_{\text {ren }}$, and $\zeta_{\text {ren }}$. The only disadvantage of this expansion is that it requires the explicit development of all the 2-point subgraphs, and also the somewhat non-trivial use of "logarithmic" power counting (see [16]).

- One might work with truncated (at the right order!) recursion relations, instead of full ones [i.e., not include the second sum in the definition (4.9) of $\delta \lambda_{k}$ ]. In the second thus obtained, only the small Mayer graphs are "partly renormalized." This is nevertheless sufficient to get the uniform geometric bounds necessary for convergence, but also requires also logarithmic power counting.

\section{The Convergence of the PRPSE}

In this section we give, under the condition (1.1) uniform estimates on the PRPSE, with all convergence rates being geometrical. It is in fact a rather straightforward application of the techniques of [16], plus some use of combinatoric arguments and of Gram's inequality similar to the detailed proof of Lemma 3.1. Therefore, we will be rather brief. The inductive bound on the amplitudes of $k$-Mayer graphs is as follows: 
We write $C^{* k}(p)=A^{* k}(p) \cdot B^{* k}(p)$ and have the following estimate to replace the sloppy bound (3.25):

$$
\sup \left(\left|A^{* k}(x, y)\right|,\left|B^{* k}(x, y)\right|\right) \leqq O(1) \cdot M^{3 k / 2} e^{-M^{k}|x-y| / 4}
$$

[the analog of (3.25) would give a bound $e^{-M^{k}|x-y| / 2 \zeta_{k}}$, which, since $\zeta_{k}$ remains bounded by 2 if $C$ in Theorem IV.1 is large enough, gives $e^{-M^{k}|x-y| / 4}$; from now on we will skip such arguments].

- There is "horizontal" exponential decay in every momentum slice $k$, between cubes of $\mathbf{D}_{k}$ in a given $k$-Mayer graph with rate corresponding to the size of the cubes of $\mathbf{D}_{k}$. This allows us to replace all the $m_{\varrho}$ dependent constants $K$ in Lemma 3.1 by true $O(1)$ constants.

- There is "vertical" exponential decay in the difference of the indices of a Mayer graph and of its external legs; this is because Mayer graphs with $N \geqq 6$ are convergent by power-counting and Mayer graphs with $N=4$ or $N=2$ are renormalized. This has been discussed at length in [16] for the Feynman graphs of any renormalizable theory, with particular applications to $\varphi_{4}^{4}$ and to $\mathrm{GN}_{2}$, so we should not repeat the analysis, but add only a few comments.

a) The gradients of the renormalization in (4.4), when integrated with respect to the fermion measure, will act on $A$ or $B$ "legs"; by translation invariance there is at most one gradient acting on each $A$ or $B$ and [see (3.33)]:

$$
\sup \left(\left|\nabla A^{* k}(x, y)\right|,\left|\nabla B^{* k}(x, y)\right|\right) \leqq O(1) \cdot M^{5 k / 2} e^{-M^{k}|x-y| / 4} .
$$

The gradients do not perturb either the power counting, or the Gram inequality. Their net effect is the gain of an exponential decrease $M^{k-j}$ between the scale $k$ of the $k$-quadruped or $k$-biped and the scale $j$ of the external leg to which the gradient applies, exactly as in [16]. By "power counting" we mean that:

$$
\left\|A^{j}\right\|_{2} \leqq O(1) M^{j / 2} ; \quad\left\|B^{j}\right\|_{2} \leqq O(1) M^{j / 2}
$$

which follows easily from the definition of $A^{j}$ and $B^{j}$. But one has also:

$$
\left\|\nabla A^{j}\right\|_{2} \leqq O(1) M^{3 j / 2} ; \quad\left\|\nabla B^{j}\right\|_{2} \leqq O(1) M^{3 j / 2} .
$$

For renormalized Mayer graphs, the Gram's structure of the determinants analogous to (3.23) is preserved, with some of the rows or columns containing derived legs $\nabla A$ or $\nabla B$ instead of $A$ and $B$. The power counting is exactly as expected, since the factor $\left(x_{j}-x_{i}\right)$ in (4.4), when multiplied by the "horizontal" exponential decrease $e^{-M^{k}\left|x_{j}-x_{i}\right|}$ of the $k$-quadruped, can be bounded by $O(1) M^{-k}$. Combining these two estimates gives a net bound $O(1) M^{j / 2} M^{-(k-j)}$, which reconstructs the normal power counting $M^{j / 2}$ of (5.1), plus the decrease $M^{-(k-j)}$ predicted, as in [16].

b) Since the cluster expansion in $\mathbf{D}_{k}$ contains both " $d / d s$ " links and links created by Mayer graphs of higher scale, one can verify that the corresponding combinatoric factors can be controlled in the same way as in the proof of Lemma 3.1; this is easy; basically the choice of the linking Mayer graphs is controlled by the "horizontal" and "vertical" decrease described above (see also [9]).

c) Finally, in the last scale the sum of the "0-super Mayer graphs" with $n$ vertices is estimated by $O(1)^{n}\left[\sup _{k}\left\{\lambda_{k}\right\}\right]^{n}$ and by Lemma 4.1 the last sum over $n$ is controlled by taking the constant $C$ in Theorem IV.1 sufficiently large. 
This general technique of estimating graphs is responsible for the bounds $O\left(\lambda_{k}^{\text {someinteger }}\right), O\left(\log k / k^{2}\right)$, etc., used in various places of this paper, like Subsect. 4.2 for instance. We will not repeat the proof in each case. We prefer to stress now the other arguments which are necessary to complete the proof of Theorem IV.1.

As remarked already, this amounts to verifying that the running parameters $\lambda_{k}^{\circ}$, $m_{k}^{\varrho}$, and $\zeta_{k}^{\rho}$ computed with the ansatz (2.21)-(2.22) and (1.1) converge to their final values $\lambda_{k}^{\infty}, m_{k}^{\infty}$, and $\zeta_{k}^{\infty}$ as $\varrho \rightarrow \infty$. This will be done by proving that these sequences are Cauchy. We can indeed compute the difference $\delta \lambda_{k}^{o} \equiv \lambda_{k}^{o+1}-\lambda_{k}^{o}, \delta m_{k}^{o}$, and $\delta \zeta_{k}^{o}$ and bound them by a summable series in $\varrho$. To achieve this let us restrict our attention for simplicity to $\delta \lambda_{k}^{\varrho}$, the other cases being similar. It is convenient to use $g_{k} \equiv\left(\lambda_{k}\right)^{-1}$ rather than $\lambda_{k}$ 's. Then pushing the analysis of (4.19) one step further (to $2^{\text {nd }}$ order in $1 / k$ ) one obtains [using (4.15) and (4.16)]:

$$
\begin{aligned}
\delta g_{k}^{\varrho}= & -(\log M)\left\{\left[\sum_{j=k+1, \ldots, \varrho} \delta^{j, \varrho}-\sum_{j=k+1, \ldots, \varrho+1} \delta^{j, \varrho+1}\right]\right. \\
& +\left[\left(\beta_{3} / \beta_{2}\right)(\log \varrho-\log (\varrho+1))-\sum_{j=k+1, \ldots, \varrho}\left(\gamma^{j, \varrho}+\delta_{3}\right) / j \beta_{2}^{j, \varrho}\right. \\
& \left.+\sum_{j=k+1, \ldots, \varrho+1}\left(\gamma_{3}^{j, \varrho+1}+\delta_{3}\right) / j \beta^{j, \varrho+1}\right] \\
& +\left[\sum_{j=k+1, \ldots, \varrho} \delta_{3} / \varrho-\sum_{j=k+1, \ldots, \varrho+1} \delta_{3} /(\varrho+1)\right]+[\text { higher orders }] .
\end{aligned}
$$

The first difference is bounded by:

$$
\sum_{j=k+1, \ldots, k-1+\varrho / 2}\left|\delta^{j, \varrho}-\delta^{j, \varrho+1}\right|+\delta^{k+\varrho / 2, \varrho+1}+\sum_{j=k+\varrho / 2, \ldots, \varrho}\left|\delta^{j, \varrho}-\delta^{j+1, \varrho+1}\right|,
$$

hence it may be bounded, using (4.16), by $O(1) \cdot e^{-O(1) \cdot \varrho / 2}$, hence by a summable series in $\varrho$. The second difference should be evaluated similarly by writing the analogue of (4.15)-(4.16) for $\gamma_{3}$ :

$$
\begin{aligned}
& \gamma_{3}^{j, \varrho}=\gamma_{3}+\delta^{j, \varrho}+O((\log j) / j) ; \quad \delta^{j, \varrho} \leqq e^{-O(1) j}+e^{-O(1)(\varrho-j)} ; \\
& \left|\delta^{j, \varrho}-\delta^{j+1, \varrho+1}\right| \leqq e^{-O(1) j} ; \quad\left|\delta^{j, \varrho}-\delta^{j, \varrho+1}\right| \leqq e^{-O(1)(\varrho-j)} .
\end{aligned}
$$

The third difference vanishes exactly. The higher orders are generated by developping the recursion relation (4.19) up to $O\left(\lambda_{k}^{2}\right)$; there is a difference

$$
\left[\sum_{j=k+1, \ldots, \varrho} \delta_{4}(\log \varrho) / \varrho^{2}-\sum_{j=k+1, \ldots, \varrho+1} \delta_{4}(\log (\varrho+1)) /(\varrho+1)^{2}\right] \simeq(\log \varrho) / \varrho^{2}
$$

coming from the $(\log \varrho) / \varrho^{2}$ correction to the $\delta_{3} / \varrho$ term in (4.19), and a remainder, $O\left(\lambda_{k}^{2}\right)$ term. The bound one can find on $\left|\delta g_{k}^{o}\right|$ without taking into account the remainder $O\left(\lambda_{k}^{2}\right)$ term in (5.2) is therefore $O(1)(\log \varrho) /(\varrho-k)^{2}$. Finally, we include in our analysis the remainder term, which is a sum of differences of terms of the form $g_{j}^{-2} \cdot f^{j, e}\left(\lambda_{j}\right)$, with $f^{j, e}\left(\lambda_{j}\right) \leqq O(1)$, by an analogue of (5.4). By an analogue of (5.5) we can also prove that $\left|f^{j, e}\left(\lambda_{j}\right)-f^{j, \varrho+1}\left(\lambda_{j}\right)\right| \leqq e^{-O(1)(\varrho-j)}$. Then we may use a simple induction. Assuming that $\left|\delta g_{j}^{\varrho}\right| \leqq O(1)(\log \varrho) /(\varrho+1-j)^{2}$ for $j=\varrho, \ldots, k+1$, 
we can bound the contribution $\left|\delta^{\prime} g_{k}^{\varrho}\right|$ to $\left|\delta g_{k}^{\varrho}\right|$ due to this term in (5.2) by:

$$
\left|\delta^{\prime} g_{k}^{\varrho}\right| \leqq O(1)\left\{\sum_{j=k+1, \ldots, \varrho}\left[\left|\delta g_{j}^{\varrho}\right| g_{j}^{-3} \cdot f^{j, \varrho}\left(\lambda_{j}\right)+g_{j}^{-2} \cdot\left|f^{j, \varrho}\left(\lambda_{j}\right)-f^{j, \varrho+1}\left(\lambda_{j}\right)\right|\right]+1 / \varrho^{2}\right\},
$$

and verify the induction hypothesis at order $k$, provided $k$ is larger than some constant integer $K$. The $K$ last steps then give just an additional $O(1)^{K}$ multiplicative factor in front of the bound for $\delta g_{k}^{o} \mid$. Since $K$ is a constant, this completes the argument to all values of $k$.

Therefore, the continuum limit of the theory exists and is computable by an absolutely convergent PRPSE expansion without cutoff and with running parameters $\lambda_{k}^{\infty}, m_{k}^{\infty}$, and $\zeta_{k}^{\infty}$.

\section{The Renormalized Expansion and Borel Summability}

In this section we will introduce the renormalized parameters in the sense of the BPHZ scheme of subtractions at 0 external momenta [18].

First let us define the renormalized quantities $\lambda_{\text {ren }}, m_{\text {ren }}$, and $\zeta_{\text {ren }}$ in terms of the Schwinger functions constructed in Theorem IV.1 by the BPHZ conditions at 0 momenta:

$$
\begin{gathered}
\lambda_{\text {ren }}=S_{4}^{T, A}(C, m)(0,0,0,0), \\
\left(m_{\mathrm{ren}}\right)^{-1}=S_{2}^{T}(C, m)(0), \\
\zeta_{\text {ren }}\left(m_{\mathrm{ren}}\right)^{-2}=\left.\left[(-i)\left(d / d p_{0}\right) S_{2}^{T}(C, m)(p)\right]_{0,0}\right|_{p=0},
\end{gathered}
$$

where $S_{4}^{T, A}\left(p_{1}, p_{2}, p_{3}, p_{4}\right)$ is the truncated amputated 4-point function at external momenta $p_{1}, p_{2}, p_{3}, p_{4}$, and $S_{2}^{T}(p)$ is the 2-point function at momentum $p$. Let us first rescale our three parameters $\lambda_{\text {ren }}, m_{\text {ren }}$, and $\zeta_{\text {ren }}$ into $\lambda_{\text {ren }} / \zeta_{\text {ren }}^{2}, m_{\text {ren }} / \zeta_{\text {ren }}$, and 1 . We obtain an equivalent two parameter family of theories, still parametrized by $C$ and $m$, but for which $\zeta_{\text {ren }}=1$. Let us call still $\lambda_{\text {ren }}$ and $m_{\text {ren }}$ (with some abuse of notations) the parameters $\lambda_{\text {ren }} / \zeta_{\text {ren }}^{2}$ and $m_{\text {ren }} / \zeta_{\text {ren }}$. Then to get the usual BPHZ renormalized series one should "invert" the map from $(C, m)$ to $\left(\lambda_{\text {ren }}, m_{\text {ren }}\right)$. (Basically, under the conditions of Theorems IV.1 and IV.2, $\lambda_{\text {ren }}$ corresponds to $C^{-1}$ and $m_{\text {ren }}$ to $m$.) We will do this in two steps. First let us reexpress the propagator in terms of the renormalized quantities $m_{\text {ren }}$ and $\zeta_{\text {ren }}=1$ rather than in terms of the running $m_{k}$ and $\zeta_{k}$. This can be done in the PRPSE, where the cluster expansion is modified so that the 2-point Mayer-graphs are defined whatever their external lines; it is also convenient to define the index of a vertex to be the second highest index of its fields. One obtains a new expansion called PRPSE' with the following rules:

- All propagators are fixed (no running masses or wave functions any more) and have the value $\left[\eta^{i}\right.$ was defined in (3.32)]:

$$
C_{\mathrm{ren}}^{i}(p)=\eta^{i}\left(p, m_{\mathrm{ren}}, \zeta_{\mathrm{ren}}\right)\left(\not p+m_{\mathrm{ren}}\right) /\left(p^{2}+m_{\mathrm{ren}}^{2}\right) .
$$


- All two-point Mayer graphs are renormalized, i.e., their amplitude at 0 momenta is subtracted, no matter how their internal indices compare to the indices of their external lines.

- A scale $k$ vertex has a coupling $\lambda_{k}$ (where now the recursion relation from $\lambda_{k+1}$ to $\lambda_{k}$ involves graphs with the renormalized propagators $C_{\text {ren }}$ instead of the $C^{* k}$ propagators.

We start by describing the expansion as it would appear if we could work directly on Feynman graphs. Consider the perturbation series of (for example) the connected Green's functions in a theory with covariance $\eta_{\rho}\left(p^{2}\right)\left(-\not p+m_{\text {ren }}\right)^{-1}$ and interaction (i.e., vertices) $\left(\lambda_{\rho}\right)(\bar{\psi} \psi)^{2}+\bar{\psi}(-\delta \zeta p) \psi-\delta m \bar{\psi} \psi$. If we choose $\delta \zeta$ and $\delta m$ correctly (the choice will of course depend on $\eta_{\rho}, m_{\text {ren }}$, and $\lambda_{\rho}$ ) we can rewrite the series as a new one with propagator $\eta_{e}\left(p^{2}\right)\left(-\not p+m_{\mathrm{ren}}\right)^{-1}$, vertices $\left(\lambda_{\ell}\right)(\psi \psi)^{2}$ (but no two-legged vertices) and having all $1 P I$ two-point functions renormalized. This is just good old BPHZ renormalization in a particularly trivial form because it is impossible to have overlapping $1 P I$ two-point subgraphs. Note that it is not necessary to know the values of $\delta \zeta$ and $\delta m$ explicitly. We now introduce a running coupling constant. Start by decomposing each propagator into a high and a low momentum part using $\eta_{\varrho}=\left(\eta_{e}-\eta_{\varrho-1}\right)+\eta_{\varrho-1}$. Next define $\delta \lambda_{\varrho}$ to be the sum of the values at zero momentum of all connected four point functions having at least three low momentum external propagators (more generally a low momentum external line or field or propagator of a subgraph is an external field whose index is lower than the ones of the internal lines). Also define $\lambda_{\varrho-1}=\lambda_{\varrho}+\delta \lambda_{\varrho}$. Next substitute $\lambda_{\varrho}=\lambda_{\varrho-1}-\delta \lambda_{\varrho}$ for the coupling constant at each low momentum vertex, i.e., at each vertex attached to at least three low momentum propagators. Each $-\delta \lambda_{\varrho}$ naturally converts its vertex into a sum of counterterms that renormalize the corresponding high momentum four point functions. This leaves us with an expansion in which all four point functions which have at least three external lines of index $\varrho-1$ are renormalized and vertices with at least three fields of index $\varrho-1$ have coupling constant $\lambda_{\varrho-1}$ (this renormalization procedure makes no problem because at scale $\varrho$ the four point functions to be renormalized cannot overlap: each one has at most one external line of index $\varrho$ ). We may now continue by decomposing $\eta_{Q-1}=\left(\eta_{Q-1}-\eta_{Q-2}\right)+\eta_{Q-2}$ and so on. The final result is an expansion in which

- the propagators are $\left(\eta_{k}-\eta_{k-1}\right)\left(-\not p+m_{\mathrm{ren}}\right)^{-1}$

- all vertices have four legs; the coupling at each vertex is $\lambda_{k}$ with $k$ being the momentum scale of the second highest line attached to the vertex

- all two point subgraphs are renormalized

- all four point subgraphs for which every internal line has momentum scale higher than the scale of the second highest external line are renormalized.

The choice we made for the renormalization of the four point function is somewhat arbitrary, it is the one which dresses as little as possible the four point function and allows a computation of the coupling constant flow $\lambda_{i-1}=\lambda_{i}+\delta \lambda_{i}$, where $\delta \lambda_{i}$ is the sum of the four point $i$-Mayer graphs (which are one particle irreducible and have at least three low momentum external fields, see below).

We must keep perturbation theory inside polymers in closed form to preserve cancellations in Gram's determinants. Therefore, to renormalize all the two point functions, even inside the polymers, requires a new development, which is again a 
sort of Mayer expansion, allowing analysis in terms of 1-particle irreducible objects instead of connected objects. We plan to give a fully detailed exposition of this expansion elsewhere $[19,9]$, but here we will give only the outline of its construction in a single momentum slice; the generalization to all slices is then similar to Sect. III.2.

We begin the description by considering those polymers that do not contain mass or wave function counterterms. We should reexpress the amplitudes of the polymers of Sect. III, to test the coupling between cubes at third order in $s_{b}$. This means that we replace $J_{b}$ in formula (3.3) by:

$$
\begin{gathered}
J_{b}=J_{b}^{1}+J_{b}^{2}+J_{b}^{3} ; \quad J_{b}^{1} F=\left.\left(d F / d s_{b}\right)\right|_{s_{b}=0} ; \\
J_{b}^{2}=\left.(1 / 2)\left(d^{2} F / d s_{b}^{2}\right)\right|_{s_{b}=0} ; \\
J_{b}^{3} F=(1 / 2) \int_{0}^{1}\left(1-s_{b}\right)^{2}\left(d^{3} F / d s_{b}^{3}\right) d s_{b} .
\end{gathered}
$$

One term in the cluster expansion (3.13) is a product of (3.8) polymers each of which is a set of squares. These squares are possibly connected by bonds $J_{b}, J_{b}^{2}$, or $J_{b}^{3}$. This term can then be viewed as a graph whose vertices are the squares, and lines are the bonds. We form a forest with

- the connected components - which have at most two low momentum fields and no true external fields - of the graph

- the 2-point subgraphs

- the 4-point subgraphs with at least 3 low momentum external fields and which are one particle irreducible.

We remove the disjointness constraints inductively in the natural order of the forest. We define for each subgraph $B$ of the forest the reduced support $S_{B}$ to be the set of all squares of $B$ that are not squares of elements of the forest contained in $B$. For example in Fig. 1 the reduced support of the subgraph $B^{\prime}$ of the graph $G$ is $S_{B^{\prime}}=\left\{\Delta_{3}, \Delta_{4}\right\}$.

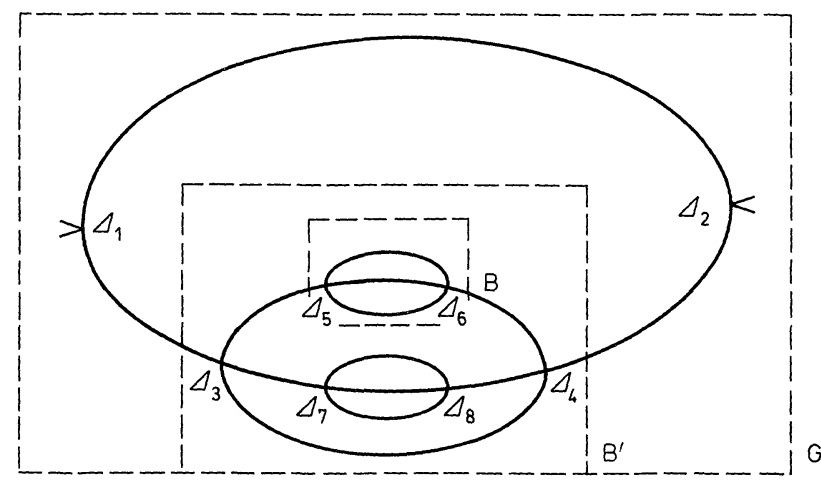

Note that every square of $G$ occurs in exactly one reduced support. We choose an order on the set of the subgraphs which is compatible with the natural order of the forest. We pick the smallest element $B_{1}$ of the forest, in that ordering, and we 
can write the constraint that its support be disjoint from the other reduced supports as:

$$
\prod_{\Delta \in S_{B_{1}}} \prod_{B^{\prime}>B_{1}} e^{-V\left(\Delta, S_{B^{\prime}}\right)},
$$

where $\prec$ is the natural ordering in the forest. These constraints may be removed by writing:

$$
\prod_{\Delta \in S_{B_{1}}} \prod_{B^{\prime} \succ B_{1}} e^{-V\left(\Delta, S_{B^{\prime}}\right)}=1+\sum_{(j, i)}\left\{e^{-V\left(\Delta_{j}, S_{i}\right)}-1\right\} \prod_{\left(j^{\prime}, i^{\prime}\right)>(j, i)} e^{-V\left(\Delta_{J^{\prime}}, S_{\imath^{\prime}}\right)},
$$

where $S_{i}=S_{B_{i}}$ and $\left\{B_{2}, \ldots, B_{n}\right\}$ is just the ordered set $\left\{B^{\prime} \mid B^{\prime}>B_{1}\right\},\left\{\Delta_{1}, \ldots, \Delta_{m}\right\}$ is some ordering on the squares of $S_{B_{1}}$, and by definition:

$$
\left(j^{\prime}, i^{\prime}\right)>(j, i) \text { if } j^{\prime}>j \text { or } j^{\prime}=j, i^{\prime}>i .
$$

We note that in the general term of the sum (6.7) there is no longer any constraint involving $\Delta_{1}, \ldots, \Delta_{j-1}$; that $\Delta_{j}$ must be contained in $S_{i}$ but not any $S_{i}$, for $i^{\prime}>i$ (but the latter constraint is redundant because $S_{i}$ must be disjoint from $S_{i}$ ) and, furthermore, that $\Delta_{j+1}, \ldots, \Delta_{n}$ must be disjoint from all $B_{2}, \ldots, B_{n}$.

In (6.7) the term containing 1 is constraint-free. We separate it into a renormalized part and two local parts. The latter are cancelled exactly by two corresponding terms, one containing a mass and the other a wave-function counterterm.

We can now move onto the next step in the induction, defining a new smaller forest for the first term of (6.7). We simply delete $B_{1}$ from the forest; for the other terms $B_{1}$ and $B_{i}$ are combined into a single element $B_{1}^{\prime}$ with reduced support $\Delta_{j+1} \cup \ldots \cup \Delta_{n} \cup S_{i}$; on this new forest we define a new order (compatible with the inclusion relation) such that if $B_{1}^{\prime}$ exists, it has the smallest possible index. We repeat the operation up to removal of all the constraints.

We may now introduce a running coupling constant as in Sect. IV. We have developed the cluster expansion up to the third order - see formula (3.4), (6.5) thus we can define the four point $k$-Mayer graphs which have only one external field of index lower than $k$; we can then renormalize them; a vertex of index $i$ with effective coupling constant is of type:

$$
\psi^{i}(x) \sum_{k \leqq i} \lambda_{k} \psi^{k}(x)\left[\psi_{k}(x)\right]^{2} .
$$

The net result is that high momentum four point functions are renormalized and we end up with an expansion similar in form to that described for ordinary perturbation theory above but expressed in terms of polymers. The only differences with the PRPSE of Sects. III and IV are that the covariances are no more effective (replace $C^{* k}$ by $C_{\text {ren }}^{k}$ ) that, the two point functions are renormalized independently of their external momenta and that the four point $k$-Mayer graphs with at least three low momentum legs are renormalized, this amounts to introducing terms of type

$$
\begin{aligned}
& {\left[\sum_{k \leqq i} \delta m_{k}\right] \psi^{i}(x) \psi_{i}(x), } \\
& {\left[\sum_{k \leqq i} \delta \zeta_{k}\right] \psi^{i}(x) \not \psi_{i}(x), } \\
& \psi^{i}(x) \psi^{j}(x)\left(\psi_{j}(x)\right)^{2} \sum_{p} \delta \lambda_{p} \quad \text { with } \quad i \geqq p \geqq j,
\end{aligned}
$$


we shall consider (6.10) as insertions in the propagator in order to get a fixed propagator (up to the finite mass) we write for the wave function term:

$$
\left[\sum_{k \leqq i} \delta \zeta_{k}\right] \psi^{i}(x) \not \psi_{i}(x)=\left[\sum_{k} \delta \zeta_{k}\right] \psi^{i}(x) \not \psi_{i}(x)-\left[\sum_{k>i} \delta \zeta_{k}\right] \psi^{i}(x) \not \psi_{i}(x) \text {. }
$$

Let

$$
\delta \zeta=\sum_{k} \delta \zeta_{k}
$$

The terms in $\delta \zeta$ reconstruct the bare propagator, i.e., the propagator between two fields of indices $i$ and $j$ is:

$$
\left[\eta^{i}(p) \delta_{i j}+\eta^{i}(p) \eta^{j}(p)\right] /\left[\left(\zeta_{\mathrm{ren}}-\delta \zeta \eta_{\varrho}(p)\right) p+\left(m_{\mathrm{ren}}\right)\right],
$$

and thus the $\beta$ 's and the $\gamma$ 's are computed with the propagator

$$
1 /\left[\left(\zeta_{\mathrm{ren}}-\delta \zeta\right) p\right]
$$

and the only wave function insertions are of type

$$
\left[\sum_{k>i} \delta \zeta_{k}\right] \psi^{i}(x) \phi \psi_{i}(x)
$$

They are the wave function insertions which were responsible for the existence of the $\delta_{3}$ term in Sect. IV.

All these insertions - (6.9), (6.11), (6.16) are small, indeed as in Sect. V we have if $\lambda_{i} \leqq \lambda_{\text {ren }} /\left(1+\lambda_{\text {ren }} i\right):$

$$
\begin{gathered}
\sum_{k \leqq i} \delta m_{k} \leqq 0(1) \sum_{k \leqq i} \lambda_{k} \leqq 0(1) \ln (k), \\
\sum_{k>i} \delta \zeta_{k} \leqq 0(1) \sum_{k>i}\left(\lambda_{k}\right)^{2} \leqq 0(1) \lambda_{i}, \\
\sum_{p, j \leqq p \leqq i} \delta \lambda_{p} \leqq 0(1) \sum_{p, j \leqq p \leqq i}\left(\lambda_{p}\right)^{2} \leqq 0(1) \lambda_{j} .
\end{gathered}
$$

This PRPSE' expansion is convergent and we have the next theorem:

Theorem VI.1. 1) For any finite values of $m_{\mathrm{ren}}, \zeta_{\mathrm{ren}}$, there exists a positive constant $C$ such that the radius of convergence $r_{\varrho}\left(m_{\mathrm{ren}}, \zeta_{\mathrm{ren}}\right)$ obeys:

$$
\left[r_{\varrho}\left(m_{\mathrm{ren}}, \zeta_{\mathrm{ren}}\right)\right]^{-1} \leqq-\beta_{2}(\log M) \varrho+\left(\beta_{3} / \beta_{2}\right) \log \varrho+C=f(\varrho, C) .
$$

Remark. Only the correct ansatz (1.1) leads to interesting non-trivial theories in the continuum as $\varrho \rightarrow \infty$; for faster decreasing choices of $\lambda_{\varrho}$ the theory will be driven to a free field as $\varrho \rightarrow \infty$.

Scheme of the Proof. The main difference with Sect. V is that $\delta \zeta$ depends on $\lambda_{0}, \ldots, \lambda_{\varrho-1}, \lambda_{\varrho}$; we proceed by induction on $\varrho, \lambda_{\text {ren }}$ being fixed, we suppose $\lambda_{0}, \ldots, \lambda_{\varrho-1}$ being computed in the theory with cutoff index $\varrho-1$ and that for $\varrho^{\prime}=\varrho-1$ :

$$
\begin{aligned}
\lambda_{i} \leqq & \left(1-O(1) \sup \left[1 / \varrho, 1 / i, e^{-\left(\varrho^{\prime}-i\right)}\right]\right) \\
& \lambda_{\text {ren }} /\left[1+\lambda_{\text {ren }}\left(i\left(-\beta_{2}\right) \ln M+\ln i \beta_{3} / \beta_{2}\right)\right] .
\end{aligned}
$$


We compute then $\lambda_{\varrho}$ by iterating $\lambda_{\varrho}=\lambda_{\varrho-1}-\delta \lambda_{\varrho}$. In this way we obtain $\lambda_{\varrho}$ of step $\varrho$ as a function of $\lambda_{0}, \ldots, \lambda_{\varrho-1}$ of step $\varrho-1$, and thus $\lambda_{0}, \ldots, \lambda_{\varrho-1}$ of step $\varrho$ and they verify (6.21) for $\varrho^{\prime}=\varrho$. It amounts just to substitute convergent series into convergent series because in $\lambda_{\varrho}$ the correction $-\delta \lambda_{\varrho}$ see $(4.14)-$ to $\lambda_{\varrho-1}$ is of second order and $\delta \lambda_{j}$ contains contributions with fields of index $\varrho$ which are in $\lambda_{j}^{2} e^{-(\varrho-j)}$.

To prove Theorem VI.1, we need only to verify that starting with $\lambda_{\varrho}$ as in (1.1), the effective constants $\lambda_{k}\left(\lambda_{\varrho}\right)$ computed from $\lambda_{\varrho}$ by the analogue of the recursion relation of Lemma 4.1 remain uniformly bounded for all $k$ by a small constant, and that the PRPSE' in these conditions is absolutely convergent. The result will then follow from analyticity of a uniform limit of analytic functions.

Assuming absolute convergence of the PRPSE', to verify that the effective constants $\lambda_{k}$ remain bounded is easy; indeed it is a simple exercise to use (4.10) to check that there is a finite constant $O(1)$ such that $\left|\lambda_{\varrho-1}\left(\lambda_{\varrho}\right)\right|$ attains its maximum in $B_{\varrho}$ for $\lambda_{\varrho}=[f(\varrho, C)]^{-1}$ if $\varrho>O(1)$. In this case we can apply Lemma 4.3 to get a bound on $\lambda_{\varrho-1}$; starting from $\varrho$ large we repeat this argument until a finite number of last recursion steps, which can be controlled by taking $C$ in Theorem IV.1 to be large enough.

It remains to check that the PRPSE' is absolutely convergent, as was the PRPSE, by Sect. V. We must show that the new Mayer expansion is convergent, and that the fact that we completely renormalize the two point function doesn't spoil the arguments of Sect. V. We can combine the "useful" part of the mass, wave function and coupling constant counterterms to get renormalized $k$-bipeds and quadrupeds as in the PRPSE, but we have additional insertions of the "useless" part of the counterterms (6.9), (6.11), and (6.16). The power counting of the wave function insertion is the same as the one of a vertex of index $i$ (because the gradient is equivalent to two fields of index $j$ ) with coefficient $\lambda_{i}$. The mass insertion is small like $i \cdot M^{-i}$. The quartic insertions are small because the coupling constant remains small. Hence the terms (6.9), (6.15), and (6.16) do not spoil the absolute convergence of the PRPSE; in short they are controlled by "asymptotic freedom," i.e., by the decay of $\lambda_{k}$ as $1 / k$, i.e., some sums over the indices are controlled by factors which have only a power decay [see (6.17)-(6.19)], each such sum "consumes" an effective coupling constant; this phenomenon is responsible for the fact that the coefficient $\beta_{3}$ in Lemma 4.2 remains $\gamma_{3}+\delta_{3}$ : the insertion of (6.15) in the graph $\longrightarrow$ gives a third order term which is the $\delta_{3}$ term.

Intuitively the absolute convergence of these expansions in which mass and wave function are fully renormalized can be summarized under the sentence that "mass and wave function, in contrast with coupling constant renormalization, do not create renormalons."

It remains to prove the convergence of the new Mayer expansion (6.6). In (6.6) there is only one sum

$$
1+\sum_{i}\left\{e^{-V\left(\Delta, S_{\imath}\right)}-1\right\} \prod_{i^{\prime}>i} e^{-V\left(\Delta, S_{\imath^{\prime}}\right)}
$$

for each square $\Delta$ of each polymer (as explained above the next sum on $\Delta$ is made 
for each term relative to the square $\Delta$ contained in $S_{i}$ ). Each sum gives a constant by square. The only difference is that $S$ can be a two point function (in Sect. III it could only be a connected component); we control the sum over $S_{i}$, i.e., the sum over the localization of $S$ using the scaled exponential decrease of the propagators of $S$ : we have to be sure that a given exponential decay is used a finite number of times. We show now that it is used at most once for this procedure.

Indeed, all the 2 point Mayer graphs which are 1 particle irreducible are graphically 2-point connected (we mean without taking in account the Mayer links $\left.\left(e^{-V\left(A, S_{i}\right)}-1\right)\right]$; in ordinary perturbation theory a propagator is graphically external to at most two 1PI graphs; however, in our procedure it can be external to more than two, because of the Mayer links: one Mayer link can link two 1PI objects $B, B^{\prime}$ which have in common a propagator with 2-point insertions giving rise to a new term $B^{\prime \prime}$, see Fig. 2; to sum on the localization of $B^{\prime \prime}$ one uses the other propagator of $B^{\prime}$.

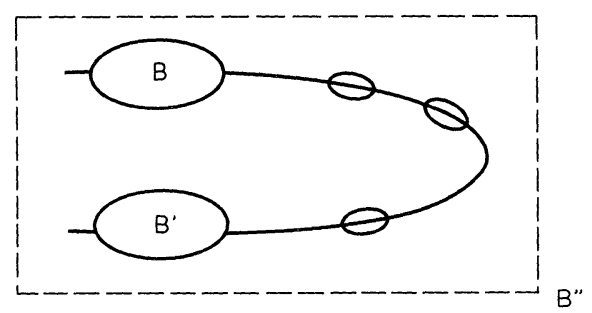

We recall that we defined the ordering on the subgraphs in such a way that a 2point Mayer graph with one propagator "used" is as small as possible; thus $B^{\prime}$ in the argument above has its two external propagators still non-used. Therefore, one of them can be used to sum on the localization of $B^{\prime \prime}$, and we conclude that each propagator is used at most once. For the 4-point function the convergence of the Mayer expansion is obvious because they have low momentum legs, and so there is no sum on their localization but only on their shape.

We are left with the verification of Borel summability (Theorem IV.2). It is done in two steps; first verify that there is analyticity in the Nevanlinna-Sokal disk $\operatorname{Re}\left(\lambda_{\text {ren }}^{-1}\right)>O(1)$ [22], then prove a "factorial bound" on the Taylor remainder at order $n$, hence the difference between the theory and the sum of the $n$ first orders of renormalized perturbation theory [22].

Since, as we remarked, $\lambda_{\text {ren }}$ is a bounded analytic function of $1 / C$, the first step consists in proving that we can extend our construction for $C$ positive and large to $\operatorname{Re} C$ positive and large. But this is again no more than an easy exercise to use the recursion relation (4.10) to check inductively that starting from $\lambda_{\varrho}^{-1}=\mathrm{f}(\varrho, \operatorname{Re} C)$ $+\lim C$ one has $\lambda_{k}^{-1}=f(k, \operatorname{Re} C)+z_{k}$, with $|\operatorname{Re} z| \leqq C / 2$.

Finally, the factorial bound on the Taylor remainder consists in studying the bound one can obtain on the PRPSE in which at most $n$ "useless" coupling constant insertions have been inserted. This problem is now well understood. It has been treated in full detail with Zimmermann's forest formalism, in [16] (see 
also [6] for a similar proof of Borel summability in $\alpha$-parametric space representation). We should not repeat again the analysis. The conclusion is that $n$ "useless" mass insertions can be bounded, paying an $n$ !; the mechanism is the usual renormalization mechanism $\sum_{k} M^{-k} k^{n} \leqq n ! O(1)^{n}$. Therefore, the Taylor remainder at order $n$ can be bounded by $n ! O(1)^{n}\left|\lambda_{\text {ren }}\right|^{n}$, which is the necessary estimate [22].

It remains to prove that the theory satisfies the O.S. axioms. Using a PauliVillars, Euclidean invariant cutoff, every axiom can be checked easily on the PRPSE' expansion except O.S. positivity (for instance the "cluster property" and existence of a mass gap is a simple consequence of the fact that $m_{0}$ is finite and nonzero. To verify O.S. positivity, we use a lattice cutoff, (identifying the corresponding theory with the former one by Borel summability, as explained in Sect. IV). The theory in a finite box can be constructed using the PRPSE'; the counterterms are then space dependent because of the volume cut-off: $\delta m$ is replaced by $\delta m(x)$ and $\delta \zeta$ by $\delta \zeta(x)$; we have then that $\delta m(x) \rightarrow \delta m, \delta \zeta(x) \rightarrow \delta \zeta$ as the cut-off tends to infinity. Now for such a theory O.S. positivity holds in the lattice, for one plane cutting the box into two symmetric parts. The infinite volume limit by translation invariance satisfies therefore the O.S. positivity.

Acknowledgements. We thank F. David for calling our attention to this model, and K. Gawezdzki and A. Kupiainen for useful discussions.

\section{References}

1. Mitter,P.K., Weisz,P.H.: Asymptotic scale invariance in a massive Thirring model with $U(n)$ symmetry. Phys. Rev. D 8, 4410 (1973)

2. Gross, D. Neveu, A.: Dynamical symmetry breaking in asymptotically free field theories. Phys. Rev. D 10, 3235 (1974)

3. Coleman, S.: Quantum sine-Gordon equation as a massive Thirring model. Phys. Rev. D 11, 2088 (1975);

Fröhlich, J., Seiler, E.: The massive Thirring-Schwinger model $\left(\mathrm{QED}_{2}\right)$ : Convergence of perturbation theory and particle structure. Helv. Phys. Acta 49, 889 (1976)

4. Feldman, J., Magnen, J., Rivasseau, V., Sénéor, R.: Massive Gross-Neveu model: A rigorous perturbative construction. Phys. Rev. Lett. 54, 1479 (1985)

5. 't Hooft, G.: On the convergence of planar diagram expansion. Commun. Math. Phys. 86, 449 (1982) and Rigorous construction of planar diagram field theories in four dimensional Euclidean space. Commun. Math. Phys. 88, 1 (1983)

6. Rivasseau, V.: Construction and Borel summability of planar 4-dimensional Euclidean field theory. Commun. Math. Phys. 95, 445 (1984)

7. Gallavotti, G., Nicolò, F.: Renormalization theory in four-dimensional scalar fields, I and II. Commun. Math. Phys. 100, 545 and 101, 247 (1985)

8. Gawedzki, K., Kupiainen, A.: Massless lattice $\phi_{4}^{4}$ theory: a nonperturbative control of a renormalizable model. Phys. Rev. Lett. 54, 92 (1985) and IHES preprint (1984)

9. Feldman, J., Magnen, J., Rivasseau, V., Sénéor, R.: "Infrared $\Phi_{4}^{4}$ ", preprint Ecole Polytechnique, and contribution to the proceedings of Les Houches summer school (1984)

10. Gawędzki, K., Kupiainen, A.: Harvard University preprint (1984)

11. Felder, G., Gallavotti, G.: Perturbation theory and non-renormalizable scalar fields. Commun. Math. Phys. 102, 549-571 (1985) 
12. Felder, G.: Work in preparation on planar $-g \varphi_{4+\varepsilon}^{4}$

13. Gawedzki, K., Kupiainen, A.: Preprints IHES and Helsinki University (1985)

14. Brydges, D.: Lectures at the 1984 "Les Houches" summer school (to be published)

15. Seiler, E.: Gauge theories as a problem of constructive quantum field theory and statistical mechanics. Lecture Notes in Physics, Vol. 159. Berlin, Heidelberg, New York: Springer 1982

16. Feldman, J., Magnen, J., Rivasseau, V., Sénéor, R.: Bounds on completely convergent Euclidean Feynman graphs. Commun. Math. Phys. 98, 273 (1985) and Bounds on renormalized graphs. Commun. Math. Phys. 100, 23 (1985)

17. Mack, G., Pordt, A.: Convergent perturbation expansions for Euclidean quantum field theory. Commun. Math. Phys. 97, 267 (1985)

18. Zimmermann, W.: Convergence of Bagoliubov's method for renormalization in momentum space. Commun. Math. Phys. 15, 208 (1969)

19. Rivasseau, V.: In preparation

20. de Calan, C., Rivasseau, V.: Local existence of the Borel transform in Euclidean $\Phi_{4}^{4}$. Commun. Math. Phys. 82, 69 (1981)

21. Epstein, H., Glaser, V.: The role of locality in perturbation theory. Ann. Inst. H. Poincare 19, $211(1973)$

22. Sokal, A.: An improvement of Watson's theorem on Borel summability. J. Math. Phys. 21, 261 (1980)

23. Wetzel, W.: Two-loop $\beta$-function for the Gross-Neveu model. Phys. Lett. 153 B, 297 (1985)

Communicated by K. Osterwalder

Received June 6, 1985; in revised form July 2, 1985

Note added in proof. After completion of this paper we realized that the optimal way to parametrize the theory is to use $\left(\lambda_{\varrho}, m_{\text {ren }}, \zeta_{\varrho}\right)$ instead of $\left(\lambda_{\varrho}, m_{\text {ren }}, \zeta_{\text {ren }}\right)$ in Sect. VI, hence to use the ansatz (1.1) for the bare coupling constant $\lambda_{\varrho}$ and fixed bare wave function constant $\zeta_{\varrho}$ and renormalized mass $m_{\text {ren }}$. This point of view will be developed in [19]. It is optimal because the renormalization flow in its asymptotic regime depends of $\zeta_{\rho}$, the value of $\zeta$ at large energy, and not of $\zeta_{\text {ren }}$. Therefore with the old parametrization we had in Sect. VI to compute $\zeta_{\varrho}$ in terms of $\zeta_{\text {ren }}$, which required the knowledge of $\lambda_{0}, \lambda_{1}, \ldots, \lambda_{\rho}$, hence a "bootstrap" inductive argument (after Theorem VI.1). This inelegant argument disappears completely with the other parametrization. (Remark that in contrast, $m_{\mathrm{ren}}$ remains a good parameter in this respect because the flow in the high energy asymptotic regime does not depend of the mass.) With the new parametrization, only Borel summability becomes slightly more complicated to check (since there are now two bare parameters to relate to the renormalized ones instead of one). 
